\title{
Broad ultra-potent neutralization of SARS-CoV-2 variants by monoclonal antibodies specific to the tip of RBD
}

\section{Running title: Potential clinical treatment to COVID-19 by SARS-CoV-2 variants}

Hang Ma ${ }^{1 *}$, Yingying Guo ${ }^{3,4 *}$, Haoneng Tang ${ }^{1 *}$, Chien-Te K. Tseng ${ }^{5,6,7,8 *}$, Lei Wang ${ }^{1}$, Huifang Zong ${ }^{1}$, Zhenyu Wang ${ }^{2}$, Yang $\mathrm{He}^{2}$, Yunsong Chang ${ }^{2}$, Shusheng Wang ${ }^{9}$, Haiqiu Huang ${ }^{9}$, Yong $\mathrm{Ke}^{1}$, Yunsheng Yuan ${ }^{1}$, Mingyuan $\mathrm{Wu}^{1}$, Yuanyuan Zhang ${ }^{3,4}$, Aleksandra Drelich $^{5}$, Kempaiah Rayavara Kempaiah ${ }^{5}$, Bi-Hung Peng ${ }^{6}$, Ailin Wang ${ }^{9}$, Kaiyong Yang ${ }^{9}$, Haiyang Yin ${ }^{1}$, Junjun Liu ${ }^{1}$, Yali Yue ${ }^{1}$, Wenbo $\mathrm{Xu}^{10}$, Shuangli Zhu ${ }^{10}$, Tianjiao $\mathrm{Ji}^{10}$, Xiaoju Zhang ${ }^{11}$, Ziqi Wang ${ }^{11}$, Gang Li ${ }^{2}$, Guangchun Liư ${ }^{2}$, Jingjing Song ${ }^{2}$, Lingling $\mathrm{Mu}^{2}$, ZongShang Xiang ${ }^{2}$, Zhangyi Song ${ }^{9}$, Hua Chen ${ }^{9}$, Yanlin Bian ${ }^{1}$, Baohong Zhang ${ }^{1}$, Hui Chen $^{1}$, Jiawei Zhang ${ }^{1}$, Yunji Liao ${ }^{1}$, Li Zhang ${ }^{9}$, Li Yang ${ }^{11}$, Yi Chen ${ }^{12}$, John Gilly ${ }^{2,9}$, Xiaodong $\mathrm{Xiao}^{2,9}$, Lei Han ${ }^{2,13 \#}$, Hua Jiang ${ }^{2,9 \#}$, Yueqing Xie ${ }^{9 \#}$, Qiang Zhou ${ }^{3,4 \#}$, Jianwei Zhu1,2,9,13\#

${ }^{1}$ Engineering Research Center of Cell and Therapeutic Antibody, Ministry of Education, China; Shanghai Jiao Tong University, Shanghai 200240, China

2Jecho Biopharmaceuticals Co., Ltd. Tianjin 300467, China

${ }^{3}$ Center for Infectious Disease Research, Westlake Laboratory of Life Sciences and Biomedicine, Key Laboratory of Structural Biology of Zhejiang, School of Life Sciences, Westlake University, Hangzhou 310024, Zhejiang, China.

${ }^{4}$ Institute of Biology, Westlake Institute for Advanced Study, Hangzhou 310024, Zhejiang, China.

5University of Texas, Medical Branch, Departments of Microbiology and Immunology, Galveston, TX 77555, USA

${ }^{6}$ nniversity of Texas, Medical Branch, Neurosciences, Cell Biology, and Anatomy, Galveston, TX 77555, USA

7University of Texas, Medical Branch, Pathology, Galveston, TX 77555, USA

¿University of Texas, Medical Branch, Center for Biodefense and Emerging Disease, Galveston, TX 77555, USA

9 Jecho Laboratories, Inc. Frederick, MD 21704, USA

${ }^{10}$ National Institute for Viral Disease Control and Prevention, China CDC, Beijing 102206, China

${ }^{11}$ Zhengzhou University People's Hospital; Henan Provincial People's Hospital, Department of Respiratory and Critical Care Medicine, Zhengzhou 450003, Henan, China 12Zhengzhou University People's Hospital; Henan Provincial People's Hospital, Clinical Research Service Center, Zhengzhou 450003, Henan, China

${ }^{13}$ Jecho Institute, Co., Ltd. Shanghai 200240, China

*These authors contributed equally: Hang Ma, Yingying Guo, Haoneng Tang, Chien-Te K. Tseng

\#Correspondent authors: LH, lei.han@jechobio.com; HJ, hua.jiang@jecholabs.com; YX, yueqing.xie@jecholabs.com; QZ, zhouqiang@westlake.edu.cn; JZ, jianweiz@sjtu.edu.cn 
45 .

\section{Abstract}

Severe acute respiratory syndrome coronavirus 2 (SARS-CoV-2) variants of concern (VOCs) continue to wreak havoc across the globe. Higher transmissibility and immunologic resistance of VOCs bring unprecedented challenges to epidemic extinguishment. Here we describe a monoclonal antibody, 2G1, that neutralizes all current VOCs and has surprising tolerance to mutations adjacent to or within its interaction epitope. Cryo-electron microscopy structure showed that $2 \mathrm{G} 1$ bound to the tip of receptor binding domain (RBD) of spike protein with small contact interface but strong hydrophobic effect, which resulted in nanomolar to sub-nanomolar affinities to spike proteins. The epitope of $2 \mathrm{G} 1$ on RBD partially overlaps with ACE2 interface, which gives $2 \mathrm{G} 1$ ability to block interaction between RBD and ACE2. The narrow binding epitope but high affinity bestow outstanding therapeutic efficacy upon $2 \mathrm{G} 1$ that neutralized VOCs with sub-nanomolar $\mathrm{IC}_{50}$ in vitro. In SARS-CoV-2 and Beta- and Delta- variant-challenged transgenic mice and rhesus macaque models, $2 \mathrm{G} 1$ protected animals from clinical illness and eliminated viral burden, without serious impact to animal safety. Mutagenesis experiments suggest that $2 \mathrm{G} 1$ could be potentially capable of dealing with emerging SARS-CoV-2 variants in future. This report characterized the therapeutic antibodies specific to the tip of spike against SARS-CoV-2 variants and highlights the potential clinical applications as well as for developing vaccine and cocktail therapy.

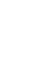

6

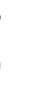

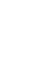

9

8

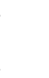

(8)

84
.

.




\section{Introduction}

Since the first Coronavirus Disease 2019 (COVID-19) case was diagnosed at the end of 2019, the severe acute respiratory syndrome coronavirus 2 (SARS-CoV-2) has caused more than 200 million confirmed infections and 4.5 million deaths in the following eighteen months, with no sign of stopping (https://ourworldindata.org/coronavirus) ${ }^{1-6}$. The hopeplaced distribution of vaccines once appeared effectively controlling the virus spread. However, the antigenic evolution of SARS-CoV-2, especially in the spike (S) protein associated with receptor binding, alters the viral immunogenicity facilitating the virus's immune escape and crossing transmission barriers ${ }^{7,8}$.

Receptor binding domain (RBD) on the $S$ protein is a determinant that mediates the binding of SARS-CoV-2 to the angiotensin-converting enzyme 2 (ACE2). Neutralizing antibodies targeting RBD were proved to be effective ${ }^{9-11}$. Correspondingly, substitutions on RBD may reduce neutralizing efficacy ${ }^{12-14}$. Several variants, listed as Variant of Concern (VOC), featured with RBD substitutions and non-RBD mutations showed to have higher transmissibility and led to more severe illness ${ }^{15-17}$, which has been causing great global dissemination concern. SARS-CoV-2 B.1.1.7 (Alpha) was first identified in United Kingdom in late summer of 2020 and rapidly became the dominant variant. This variant has nine mutations in the $\mathrm{S}$ protein, one of which is $\mathrm{N} 501 \mathrm{Y}$ in $\mathrm{RBD}^{18}$. Alpha variant possesses a comparative transmission advantage, with a reproductive number $50 \%$ to $100 \%$ higher than other non-VOC lineages ${ }^{1}$. Vaccine-elicited neutralizing antibody responses were shown to be at risk of being desensitized by Alpha ${ }^{19}$. SARS-CoV-2 B.1.351 (Beta) has three substitutions in RBD, i.e., K417N, E484K, and N501Y. Incorporation of E484K empowers variants possible being completely resistant to plasma neutralization ${ }^{20}$. Mutations E484K together with $\mathrm{K} 417 \mathrm{~N}$ and N501Y largely contribute to the escape of Beta variant from convalescent and vaccine-induced sera ${ }^{21,22}$. SARS-CoV-2 P.1 (Gamma) shares three identical site-mutations in RBD with Beta variant, and their differences are that the substitution of K417 is threonine in Gamma variant, while is asparagine in Beta variant. Similarly, Gamma variant notably reduced susceptibility to antibody treatment and vaccine protection $^{23,24}$. SARS-CoV-2 B.1.617.2 (Delta) was first reported in India and quickly spread globally in the first half of 2021. This strain has more than ten $S$ protein mutations and two of them, L452R and T478K, are in RBD. Delta variant exhibits more extensive immunologic resistance than Alpha, escaping from many $S$ protein antibodies targeting RBD and non-RBD epitopes ${ }^{25,26}$. Individuals who recovered from Beta and Gamma variants are more susceptible to be infected with Delta ${ }^{27}$. In addition to these VOCs, potential outbreaks of several variants have raised public concern, such as the recently rapidly spreading variant C.37 (Lambda) ${ }^{28}$ and the new variant B.1.621 (Mu) ${ }^{29}$. The emergence of these variants, even possible hybrid variants, raises the risk of compromising the therapeutic effectiveness of vaccines and neutralizing antibodies that were previously developed ${ }^{30,31}$.

Here we report our efforts on discovering neutralizing antibodies that provide extensive protection against the variants with global impact, especially the VOCs. We isolated RBD positive single $B$ cells from convalescent individuals and cloned monoclonal antibodies (mAbs) within. After a series of programmed screening, several antibodies with remarkable neutralizing effect were panned out from the candidates (Fig. 1a). One of these antibodies, 
designated as 2G1, efficiently neutralized all VOCs including widely spread Alpha, Beta, Gamma, Delta variants and Cluster 5 , a variant with Y453F substitution once caused public concern due to the zoonotic characteristics. The antibody $2 \mathrm{G} 1$ was subsequently fully characterized physic-chemically and biologically, as well as evaluated in potential in clinical applications.

\section{Results}

Molecule discovery of $2 \mathrm{G} 1$

We collected blood samples from 20 convalescent individuals who were infected by SARSCoV-2 in February 2020. Peripheral blood mononuclear cells were enriched and sorted with fluorescently labeled recombinant SARS-CoV-2 RBD (WA1/2020) protein (Fig. 1b). Over $1200 \mathrm{~B}$ cells were isolated and more than 500 pairs of $\lg \mathrm{G}$ antibody genes were cloned by single-cell PCR. Of which, 375 are kappa subtype and 174 are lambda subtype (Fig. 1c). 143 RBD binders were obtained after the ELISA-based preliminary screening (Fig. 1d). In the following pseudovirus-based screening, three molecules, including 2G1, displayed ultra-potent neutralization with $\mathrm{IC}_{50}$ less than $0.01 \mu \mathrm{g} / \mathrm{mL}$ (Fig. 1e). Antibody $2 \mathrm{G} 1$ stood out from these candidates after further investigation despite the binding and ACE2 blocking abilities were not remarkable (Supplementary information, Fig. S1a-b). In the germline analysis of 33 candidates, 23 heavy chains were from IGHV3 and 18 light chains were from IGKV1 (Supplementary information, Fig. S2). Six heavy chains, including 2G1, were from IGHV3-53, which was reported having short complementarity-determining region and with minimal affinity but high efficacy ${ }^{32}$.

WA1/2020 RBD-mFc and S trimer proteins and pseudovirus were employed to further confirm the antigen-binding and neutralizing ability of 2G1. Antibody $2 \mathrm{G} 1$ bound to RBD$\mathrm{mFc}$ and S trimer with $\mathrm{EC}_{50}$ of $0.016 \mu \mathrm{g} / \mathrm{mL}$ and $0.135 \mu \mathrm{g} / \mathrm{mL}$ (Fig. 2a-b) and neutralized WA1/2020 pseudovirus with $\mathrm{IC}_{50} 0.0031 \mu \mathrm{g} / \mathrm{mL}$ (Fig. 2c), in line with the results of previous screening. Affinity of monovalent $2 \mathrm{G} 1$ (Fab) to RBD was measured by surface plasmon resonance (SPR). Relatively moderate dissociation constant $\left(K_{d}\right)$ of $2 G 1$ to WA1/2020 RBD was determined as $1.05 \times 10^{-3} \mathrm{~s}^{-1}$. The rapid binding of $2 \mathrm{G} 1$ with association constant $\mathrm{K}_{\mathrm{a}}$ $=2.55 \times 10^{6} \mathrm{Ms}^{-1}$ offered a sub-nanomolar equilibrium dissociation constant $\left(\mathrm{K}_{\mathrm{D}}\right)$ value of $0.41 \mathrm{nM}$ (Fig. 2d). Next, the antibody 2G1 was moved to further characterization including in vitro and in vivo biological activities as well as structural and mechanism investigation.

\section{G1 neutralizing SARS-CoV-2 variants}

With the continuing spread of mutations, combating SARS-CoV-2 variants has become a crucial task. We explored the effects of $2 \mathrm{G} 1$ on the mutations at several important sites such as N439K, Y453F, E484K and N501Y in terms of blocking the ACE2-RBD interaction. The $\mathrm{IC}_{50}$ of $2 \mathrm{G} 1$ blocking WA1/2020 RBD, N439K, Y453F, E484K and N501Y interacting with ACE2 were $0.1504,0.1050,0.2225,0.1951$ and $0.1672 \mu \mathrm{g} / \mathrm{mL}$, respectively (Fig. 3a). To further study the $S$ mutants of VOCs influence on blocking ability of $2 \mathrm{G} 1$, mutant trimeric $S$ proteins of VOCs were used in ACE2 blocking experiment. The $\mathrm{IC}_{50}$ of $2 \mathrm{G} 1$ were 0.0821 , $0.1066,0.1074,0.1047$, and $0.7973 \mu \mathrm{g} / \mathrm{mL}$, corresponding to WA1/2020, Alpha, Beta, Gamma, and Delta (Fig. 3b). We determined the affinities of $2 \mathrm{G} 1$ with various $S$ trimers using SPR. 2G1 Fab bound to $S$ trimers with nanomolar affinities. $K_{D}$ of its binding to 
WA1/2020, Alpha, Beta, Gamma, Kappa, and Delta were 1.02, 0.86, 2.77, 2.30, 1.04, and $15.30 \mathrm{nM}$, respectively (Fig. 3c). The dissociation rate of 2G1/Delta $\left(\mathrm{K}_{d}=4.27 \times 10^{-2} \mathrm{~s}^{-1}\right)$ was increased as compared with WA1/2020 $\left(\mathrm{K}_{d}=1.05 \times 10^{-3} \mathrm{~s}^{-1}\right)$, which leads to the decrease in affinity.

In pseudovirus neutralization assays, we found that antibody $2 \mathrm{G} 1$ robustly neutralized all pseudoviruses, including D614G, Alpha, Beta, Gamma, Delta, and Cluster 5 variants (Fig. 4a-g, Supplementary information, Fig. S3) with low $\mathrm{IC}_{50}$, especially $0.0005 \mu \mathrm{g} / \mathrm{mL}$ against Gamma and $0.0002 \mu \mathrm{g} / \mathrm{mL}$ against Cluster 5. Live SARS-CoV-2 neutralization assay results were consistent with those from experiments using pseudoviruses. Antibody 2G1 neutralized WA1/2020 live virus with $\mathrm{IC}_{50}$ of $0.0240 \mu \mathrm{g} / \mathrm{mL}$ (Fig. $4 \mathrm{~h}$ ) while it was more inclined to neutralize Alpha, Beta, and Gamma live virus, with $\mathrm{IC}_{50}$ decrease about 1.7-fold $(0.0138 \mu \mathrm{g} / \mathrm{mL}), 5.2$-fold $(0.0046 \mu \mathrm{g} / \mathrm{mL})$, and 3.0-fold $(0.0079 \mu \mathrm{g} / \mathrm{mL})$. In this assay, $2 \mathrm{G} 1$ had the same neutralizing activity $\left(\mathrm{IC}_{50}=0.0240 \mu \mathrm{g} / \mathrm{mL}\right)$ against Delta and WA1/2020.

In vivo protection in animal models

To evaluate in vivo antiviral efficacy of $2 \mathrm{G} 1$ against SARS-CoV-2 challenge, we performed viral clearance assay employing both ACE2 transgenic mouse and rhesus macaque models. In the transgenic mouse study, animals were challenged with high copies of 100 times of half lethal dose (LD $\left.{ }_{50}\right)$ of SARS-CoV-2 WA1/2020, Beta, or Delta at day 0 , followed by three different $2 \mathrm{G} 1$ dose treatments $(20,6.7$ or $2.2 \mathrm{mg} / \mathrm{kg}$ ) or vehicle injection (PBS). Four days post infection (dpi), four mice in each group including vehicle and differentially treated groups were euthanized, and lungs and brains were collected for the titration of viral load (Fig. 5a). Mice treated with vehicle developed an acute wasting syndrome and quickly met the designed endpoint at $5 \mathrm{dpi}$. In contrast, WA1/2020 and Beta virus-infected mice that received $20,6.7$ or $2.2 \mathrm{mg} / \mathrm{kg}$ treatments survived without losing any weight or revealing any obvious signs of illness throughout the study (Fig. 5b-d). Delta virus-infected mice in the $20 \mathrm{mg} / \mathrm{kg}$ group all survived throughout the trial period and had a good clinical wellbeing score. In the same study, $55.6 \%$ mice in the $6.7 \mathrm{mg} / \mathrm{kg}$ group and $10 \%$ mice in the $2.2 \mathrm{mg} / \mathrm{kg}$ group recovered back to healthy physiological condition (Fig. $5 \mathrm{~b}-\mathrm{d}$ ) from the virus challenge. The results indicated that at the range of $6.7-20 \mathrm{mg} / \mathrm{kg} 2 \mathrm{G} 1$ antibody treatment was effective for animals to recover from the viral infection.

In the study of rhesus macaque animal model (Fig. 6a), the animals were infected with $10^{5}$ TCID $_{50}$ of SARS-CoV-2 (2019-nCoV-WIV04) per animal and randomly divided into control (vehicle injection), low-dose (10 mg/kg of $2 \mathrm{G} 1$ ), and high-dose (50 mg/kg of 2G1) groups, with one male and one female in each group. Drugs were intravenously given $24 \mathrm{~h}$ post infection. All animals in the two therapy groups had a high viral load of $10^{6}$ copies $/ \mathrm{mL}$ in the throat swab at $1 \mathrm{dpi}$. After the drug injection, the viral titer was gradually decreased. The throat virus was cleared at $3 \mathrm{dpi}$ in one of the high-dose animals and at $4 \mathrm{dpi}$ in the remaining treated animals (Fig. 6b). One animal in the control group had an elevated viral titer in the anal swab at $5 \mathrm{dpi}$, but no animals in the antibody treated groups showed this trend until 7 dpi (Fig. 6c). In addition, we checked the viral distribution in lung, trachea, and bronchus tissues. The virus was detectable in most areas of the lungs, in the tracheas, and bronchi of the control animals. In the group treated with high-dose of the antibody, the virus was present in right-middle, left-middle, and left-lower of the lungs, as well as left-bronchi. 
221 In the low-dose group, the virus was only found in tracheas (Fig. 6d). Results from both transgenic mouse and rhesus macaque studies showed a promising protective efficacy of $2 \mathrm{G} 1$, in consistent with the in vitro neutralization results.

We further investigated the Fc effector function of 2G1. Results showed that $2 \mathrm{G} 1$ had no obvious antibody-dependent cellular cytotoxicity (ADCC) effect (not shown) but moderate antibody-dependent cellular phagocytosis (ADCP) up to $35 \%$ (Supplementary information, Fig. S4a). We hypothesize that the moderate ADCP may help the antigen presentation of SARS-CoV-2. Pharmacokinetics (PK) study revealed the half-life of 2G1 in mice was 11.1 days (Supplementary information, Fig. S4b), similar to many therapeutic antibodies. Mice treated with $15 \mathrm{mg} / \mathrm{kg}, 30 \mathrm{mg} / \mathrm{kg}$, or $60 \mathrm{mg} / \mathrm{kg}$ showed no statistical changes in body weight, white blood cell count, red blood cell count, hemoglobin, and platelets (Supplementary information, Fig. S4c-g). Mice received 2G1 treatment had no evident pathological changes in hearts, livers, spleens, lungs and kidneys (Supplementary information, Fig. S5). Currently, Investigational New Drug-directed systematic safety assessment is ongoing to support the pre-clinical safety of using $2 \mathrm{G} 1$ in human clinical trials. Toxicology study in nonhuman primate showed that $2 \mathrm{G} 1$ was well tolerated at the maximum experimented dosage of $200 \mathrm{mg} / \mathrm{kg}$.

\section{Cryo-EM structure of the complex between $2 \mathrm{G} 1$ and SARS-CoV-2 S protein}

To investigate the binding mode of antibody $2 \mathrm{G} 1$ on $S$ trimer, we solved the cryo-electron microscopy (cryo-EM) structure of $2 \mathrm{G} 1$ in complex with $\mathrm{S}$ trimer at $2.7 \AA$ resolution (Fig. 7a, Supplementary information, Fig. S6-7). Yet, the cryo-EM map density on the interface between RBD and $2 \mathrm{G} 1$ were smeared. So, we performed local refinement and improved the antibody-antigen interface resolution to $3.2 \AA$, enabling reliable analysis of the interactions between the RBD and 2G1 (Fig. $7 \mathrm{~b}$ ). In the S/2G1 complex, three solved Fabs bound to trimeric $S$ with all RBDs in the "down" position and the $S$ protein in a locked conformation ${ }^{33,34}$ (Fig. 7a). There is an additional density in RBD domain of the structure, which was reported as free fatty acid linoleic acid (LA) in a locked conformation ${ }^{33}$.

For detailed analysis of the interface, antibody $2 \mathrm{G} 1$ binds to tip area of RBD of $S$ trimer, overlapping with the ACE2 binding site on RBD and offset from the major mutational hotspots in VOCs. The heavy chain of $2 \mathrm{G} 1$ interacts with RBD mainly through three complementarity-determining regions, named CDRH1 (residues 30 to 35), $\mathrm{CDRH} 2$ (residues 50 to 65), and CDRH3 (residues 98 to 111). The light chain of 2G1 participates interaction mainly through two CDRs, CDRL1 (residues 23 to 36) and CDRL3 (residues 91 to 100 ) (Fig. $7 \mathrm{~b}-\mathrm{e}$ ). The interface between RBD and 2G1 is stabilized by an extensive hydrophobic interaction network. Phe486 on the RBD top loop interacts with Tyr33, Tyr52 on heavy chain and Tyr34, Tyr93, Trp99 on light chain through hydrophobic and/or $\pi-\pi$ interactions simultaneously (Fig. $7 \mathrm{c}$ ). $\mathrm{CDRH} 1$ and $\mathrm{CDRH} 3$ of the $2 \mathrm{G} 1$ heavy chain were positioned above the LA binding pocket in the adjacent RBD' (Fig. 7b and 7e). We further compared 2G1 with three antibodies (S2E12, B1-182.1 and REGN10933), which have similar patterns of epitope (Fig. 8a-C). Structural comparison reveals that the epitope for 2G1 partially overlaps with these three antibodies (S2E12, B1-182.1 and REGN10933), but they have different binding directions (Fig. 8b). Besides, 2G1 has a relative narrow binding epitope which may result less probability of losing neutralizing activity due to viral 
mutagenesis (Fig. 8c).

Potential escape risk evaluation

To address the potential virus escape issue, we collected the high-frequency mutation sites near the 2G1 binding epitope from GISAID database as of August 2021 (Fig. 9a), and constructed a series of $S$ protein sequences containing these mutations. The change in binding ability of $2 \mathrm{G} 1$ was reflected by the normalized mean fluorescent intensity (MFI) relative to the wild-type $S$ protein in flow cytometry. Mutants $484 \mathrm{~K}, 477 \mathrm{~N} / 484 \mathrm{Q} / 490 \mathrm{~S}$, and 477R/478K/484K distinctly reduced $2 \mathrm{G} 1$ binding (Fig. 9b). Mutants 477N/490S, 477R/490S, 478K/484Q, and 484K/490S remarkably enhanced $2 \mathrm{G} 1$ binding (Fig. 9b). The $484 \mathrm{~K}$ substitution is featured in variants Beta and Gamma. Although $484 \mathrm{~K}$ alone leads to a decreased binding ability of $2 \mathrm{G} 1$, trimeric $S$ harbor all mutation sites only slightly influenced the affinity of $2 \mathrm{G} 1$ (Fig. 3c). The 484K substitution leads to the loss of salt bridge between Glu484 and ACE2 Lys31, resulting in the reduced affinity of ACE2 ${ }^{35}$. It may be one of the reasons why the activity of $2 \mathrm{G} 1$ even slightly improved in neutralizing Beta and Gamma mutants. Another substitution in residue 484 with Gln (484Q) only slightly weakened the binding of 2G1 (Fig. 9b). SARS-CoV-2 Delta variant possesses the T478K substitution, which is a contact residue with $2 \mathrm{G} 1$. The single point mutation with T478K has mildly decreased the $2 \mathrm{G} 1$ binding (Fig. 9b), which is consistent with the SPR data.

We also directly mutated the key interacting residues between RBD and $2 \mathrm{G} 1$ by alanine substitution, though they are not high-frequency mutation sites. Only moderate decline in $2 \mathrm{G} 1$ interaction was found in several mutations, including 486A, 489A, 477A/487A, and 477A/489A (Fig. 9c). These results suggest that $2 \mathrm{G} 1$ could potentially be effective against future SARS-CoV-2 variants.

\section{Discussion}

SARS-CoV-2 has no sign of stopping its transmission since the outbreak, and the emergence of variants with increased transmissibility and capability of surveillance escape has assisted its continued existence. Recently, the variant Delta has become an intensively concerned strain due to its unparalleled transmissibility, which is embodied in the 1000 times higher viral load than the ancestral strain of SARS-CoV-26,36. The high-frequency mutation nature of SARS-CoV-2 necessitates the development of therapies with breadth $^{37,38}$. We screened antibodies with broad spectrum of neutralizing effects from convalescent subjects. One of which, 2G1, showed excellent and extensive neutralization

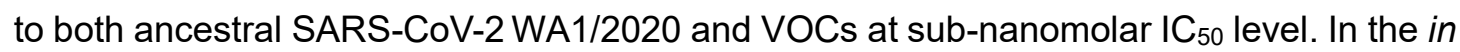
vivo study, transgenic mice infected by the WA1/2020- and Beta- were cured by antibody $2 \mathrm{G} 1$ at a dose as low as $2.2 \mathrm{mg} / \mathrm{kg}$, as well as fully protected from Delta infection in the range from 6.7 to $20 \mathrm{mg} / \mathrm{kg}$, even when animals were challenged with 100 times $L D_{50}$ of viral load. These results indicate that $2 \mathrm{G} 1$ is a potent therapeutic antibody against the broad spectrum of variants currently being concerned.

The cryo-EM structure of $2 \mathrm{G} 1$ in complex with the $S$ protein revealed that $2 \mathrm{G} 1$ binds to the tip of $S$ trimer through small interface but strong hydrophobic effect. The strong hydrophobic effect provides high affinity for $2 \mathrm{G} 1$, and the $\mathrm{K}_{\mathrm{D}}$ of interaction with $\mathrm{S}$ trimers of SARS-CoV-2 and VOCs ranges from $0.86 \mathrm{nM}$ to $15.3 \mathrm{nM}$. SARS-CoV-2 variants Beta and 
Gamma possess E484K and N501Y substitutions, which are adjacent to the epitope of $2 \mathrm{G} 1$. We correspondingly detected a slight decrease in the affinity of $2 \mathrm{G} 1$, from $1.02 \mathrm{nM}$ for WA1/2020 to $2.77 \mathrm{nM}$ for Beta and $2.30 \mathrm{nM}$ for Gamma. Surprisingly, $2 \mathrm{G} 1$ showed no compromise in activity against Beta and Gamma in both pseudo- and live- viruses, and both in vitro and in vivo. The dose of $2.2 \mathrm{mg} / \mathrm{kg}$ of $2 \mathrm{G} 1$ completely cleared the viral load in Beta virus challenged transgenic mice, and the efficacy of which was as good as for WA1/2020 virus challenged mice. The $\mathrm{IC}_{50}$ even improved in the in vitro live virus test, decreased from $0.0240 \mu \mathrm{g} / \mathrm{mL}$ against WA1/2020 to $0.0046 \mu \mathrm{g} / \mathrm{mL}$ against Beta and 0.0079 $\mu \mathrm{g} / \mathrm{mL}$ against Gamma. These results suggest that changes in affinity may not ultimately determine the therapeutic effect of neutralizing antibodies, and various other factors could be involved ${ }^{35,39}$. In addition, the small binding epitope reduces the probability of interference between $2 \mathrm{G} 1$ and other RBD antibodies so that $2 \mathrm{G} 1$ can cooperatively work with those antibodies to achieve a synergistic effect, for better responding to immunologic evasion of SARS-CoV-2 variants.

Furthermore, the specific $2 \mathrm{G} 1$ antibody epitope of RBD tip is offset from mutational hot spots and increases neutralization breadth covering new-onset VOCs. Variants Lambda comprising L452Q/F490S and Mu comprising E484K/N501Y in RBD have recently raised concerns $^{28,29}$. Although residue 490 is nearing $2 \mathrm{G} 1$ epitope, our results suggested that F490S did not cause significant affinity alteration. The E484K/N501Y substitution in variant $\mathrm{Mu}$ is also seen in Beta and Gamma. In view of the good binding and neutralization of 2G1 against Beta and Gamma, we believe that $2 \mathrm{G} 1$ will likely be comparatively effective against $\mathrm{Mu}$. In addition, we directly mutated the amino acid residues adjacent to the epitope on RBD by $2 \mathrm{G} 1$, as well as several residues that directly interact with $2 \mathrm{G} 1$, and found that only few mutation groups may cause a significant weakening of the $2 \mathrm{G} 1$ binding ability. Collectively, the model of $2 \mathrm{G} 1$ binding to the tip of $S$ trimer provides a good reference for developing vaccines and optimizing a better combination therapy.

The neutralizing antibody 2G1 has been manufactured under cGMP to support the Investigational New Drug application. We would believe that antibody treatment with 2G1 will bring clinical benefit to COVID-19 patients.

\section{Materials and Methods}

B cells

Blood samples were obtained from patients who were recovered from COVID-19 for 10 weeks and had a negative nucleic acid test. Samples with serum antibody titer over $1 \times$ $10^{6}$ were chosen for the peripheral blood mononuclear cells (PBMCs) separation using Ficoll density gradient centrifugation method. B cells were enriched applying a human B Cell Isolation Kit (Stemcell). Afterwards, B cells were then stained with APC-Alex700 labeled anti-CD19 (BD), BV421 labeled anti-CD27 (BD), BV510 labeled anti-lgG (BD), Biotin labeled RBD (Sino Biological), PE labeled streptavidin (ThermoFisher) and 7AAD (BD) Single memory B cells with potential SARS-CoV-2 antibody secretion were sorted out by gating $7 A A D^{-}, C D 19^{+}, C D 27^{+}, \operatorname{lgG}{ }^{+}$, and $\mathrm{RBD}^{+}$using a $\mathrm{BD}$ Aria III cell sorter with fluorescence-activated cell sorting modules. $B$ cells were suspended into lysis buffer and quickly frozen. B cell mRNA was subsequently converted to cDNA by SuperScript III Reverse Transcriptase (Invitrogen) and $V$ gene were rescued by PCR. Linear Cassettes 
were composed of $C M V$ promoter $V_{H}$ or $V_{L}$ and polyA tail, and were used for expressing a small amount of antibody for preliminary screening.

mAb preparation

Heavy chains and light chain genes were inserted separately into pcDNA3.4 and amplified in E. coli DH5a. PureLink ${ }^{\mathrm{TM}}$ HiPure Plasmid Miniprep Kit (Invitrogen) was used for low endotoxin plasmid preparation. Monoclonal antibodies were transiently expressed by cotransfecting ExpiCHO-S cells (ThermoFisher) with heavy chain and light chain plasmids using an ExpiCHO ${ }^{\mathrm{TM}}$ Expression System (Gibco). Cell culture was harvested after an 8- to 14- day incubation at $37^{\circ} \mathrm{C}$ with humidified atmosphere of $8 \% \mathrm{CO}_{2}$ with shaking. Full-length IgG was obtained by affinity purification utilizing a Protein A chromatography column (GE Healthcare) in AKTA avant (Cytiva). For long-term storage, antibodies were kept in a solution containing $10 \mathrm{mM}$ Histidine- $\mathrm{HCl}, 9 \%$ trehalose, and $0.01 \%$ polysorbate 80 .

\section{T-ACE2 cells}

To obtain HEK-293T cells with stable expression of ACE2 protein, a lentiviral system bearing ACE2 (Genbank ID: BAJ21180.1) gene was constructed. In brief, HEK-293T cells (ATCC) with $70 \%-80 \%$ confluence in a $10 \mathrm{~cm}$ dish were co-transfected with $12 \mu \mathrm{g}$ of plasmid pHIV-puro encoding RRE and ACE2 genes, $8 \mu \mathrm{g}$ of plasmid psPAX2 encoding gag and pol, and $4 \mu \mathrm{g}$ of plasmid VSV-G encoding $\mathrm{G}$ glycoprotein of vesicular stomatitis virus(VSVG) using Lipofectamine 3000 Reagent (Invitrogen). $12 \mathrm{~h}$ later, the medium was changed to fresh DMEM (Gibco) supplemented with 10\% FBS (Gibco) for another $48 \mathrm{~h}$ culturing. Medium containing virus particles was harvested and concentrated using a Lentivirus Concentration Kit (Genomeditech). The concentrated virus particles were used to infect HEK-293T cells under selection pressure of $10 \mu \mathrm{g} / \mathrm{mL}$ puromycin (Beyotime Biotechnology). The transfection efficiency was examined by flow cytometry using S1-mFc recombinant protein (Sino Biological) as primary antibody and FITC-AffiniPure Goat AntiMouse IgG (Jackson) as secondary antibody. The resulting bulk transfected population was sorted on a BD FACSJazz Cell Sorter (BD) with the BD FACS ${ }^{\mathrm{TM}}$ Sortware. Cells with top $1 \%$ fluorescence intensity were retained and expanded for subsequent use.

\section{S protein over-expression cells}

The coding sequence for full-length wild-type S protein (GenBank: QHD43416.1) from Met1 to Thr1273 was inserted into plasmid pHIV-puro1.0, followed by an internal ribosome entry site (IRES) and puromycin resistance gene. The lentiviruses were generated using the HEK-293T packaging system as mentioned above. $500 \mu \mathrm{L}$ of filtered lentivirus supernatant was added in a 24-well plate with Jurkat cells (ATCC). After cell expansion and selection with $10 \mu \mathrm{g} / \mathrm{mL}$ puromycin for one week, the positive $S$ expression was confirmed by flow cytometry.

\section{Antigen-binding ELISA}

Enzyme-linked immunosorbent assays (ELISA) were applied to study the binding ability of antibodies with SARS-CoV-2 RBDs (Sino Biological) and S trimers (AcroBiosystems). Antigens were diluted with ELISA Coating Buffer (Solarbio) to $1.0 \mu \mathrm{g} / \mathrm{mL}$ and immobilized 
onto High Binding ELISA 96-Well Plate (BEAVER) with $100 \mu \mathrm{L}$ per well overnight at $4^{\circ} \mathrm{C}$. Plates were washed 4 times with PBST (Solarbio) and blocked with $3 \%$ skim milk for $1 \mathrm{~h}$ at $37^{\circ} \mathrm{C}$. Then, serially diluted antibodies were added $100 \mu \mathrm{L}$ per well and incubated at $37^{\circ} \mathrm{C}$ for $1 \mathrm{~h}$. After pipetting off the unbound antibodies, plates were washed 4 times with PBST and further incubated with $100 \mu \mathrm{L}$ per well of goat anti-human IgG (Fc specific)-Peroxidase antibody ( $1: 5000$ dilution, Sigma) for $1 \mathrm{~h}$ at $37^{\circ} \mathrm{C}$. After a final 4 times washing with PBST, the binding of antibodies with SARS-CoV-2 antigens were visualized by adding $100 \mu \mathrm{L}$ peroxidase substrate TMB Single-Component Substrate solution (Solarbio) and incubating for $15 \mathrm{~min}$ in dark. The reaction was terminated by adding $50 \mu \mathrm{L}$ stop buffer (Solarbio) and the plates were immediately submitted to an ELISA microplate reader (TECAN Infinite M200 Pro) to measure the optical density (OD) at $450 \mathrm{~nm}$. Data were analyzed with GraphPad Prism Version 9.0.0 and $\mathrm{EC}_{50}$ values were determined using a four-parameter nonlinear regression.

\section{ACE2 competition ELISA}

For experiments involving the competitive binding of antibodies to SARS-CoV-2 RBD or S trimer, recombinant hACE2-Fc protein was first biotinylated using EZ-Link Sulfo-NHSBiotin (ThermoFisher) as the instruction described. SARS-CoV-2 RBD (Sino Biological), S trimer (AcroBiosystems), mutated RBDs (Sino Biological), and mutated $S$ trimers (AcroBiosystems) were coated onto High Binding ELISA 96-Well Plate (BEAVER). In order to obtain an optimized hACE2-Fc concentration for this experiment, the concentrationdependent binding of biotinylated hACE2-Fc to coated SARS-CoV-2 antigens was measured by performing a conventional receptor-binding ELISA. The $\mathrm{EC}_{80}$ of biotinylated hACE2-Fc was calculated by the four-parameter nonlinear fitting. Antibodies were serially diluted in 1\% BSA (Sigma) and added $50 \mu \mathrm{L}$ into the antigen coated plates. Biotinylated hACE2-Fc at EC 80 concentration was subsequently pipetted into. After incubation at $37^{\circ} \mathrm{C}$ for $1 \mathrm{~h}$, plates were 4 times washed with PBST and incubated with $100 \mu \mathrm{L}$ of $1: 2000$ diluted Ultrasensitive Streptavidin-Peroxidase Polymer (Sigma). After further washing, 100 $\mu \mathrm{L}$ TMB was added, and followed by detection of the bound hACE2 in the microplate reader. Four-parameter nonlinear regression fitting in GraphPad Prism Version 9.0.0 was applied for result analysis.

\section{Surface Plasmon Resonance (SPR)}

The binding affinities of antibodies to SARS-CoV-2 RBD and $S$ trimers (wildtype/B.1.1.7/B.1.351/P.1/B.1.617.1/B.1.617.2) were tested using a BIAcore 8K system (Cytiva) together with CM5 biosensor chips (Cytiva). Antigens were diluted in pH 5.0 Acetate (Cytiva) and covalently coupled on chips using an Amine Coupling Kit (Cytiva). After reaching a $70 \mathrm{RU}$ coupling level, the excess antigens were washed away and the unbound sites were blocked with ethanolamine. Antibodies were 2-fold serially diluted from $1.250 \mu \mathrm{g} / \mathrm{mL}$ to $0.039 \mu \mathrm{g} / \mathrm{mL}$ in HBS-EP buffer (Cytiva) and then injected for $120 \mathrm{~s}$ at 30 $\mu \mathrm{L} / \mathrm{min}$. After that, the binding was dissociated with HBS-EP buffer for $120 \mathrm{~s}$, followed by chip regeneration with $\mathrm{pH} 1.5$ Glycine (Cytiva). Parameters including $\mathrm{K}_{\mathrm{a}}, \mathrm{K}_{d}$ and $\mathrm{K}_{\mathrm{D}}$ values were calculated employing a monovalent analyte model with BIAevaluation software. 
Pseudovirus neutralization

ACE2-293T cells were seeded in a white 96-well plate (Corning) at a density of $1 \times 10^{4}$ cells per well one night prior to use. Serially diluted antibodies were incubated with wildtype (Yeasen) or mutant pseudoviruses (GENEWIZ) for $0.5 \mathrm{~h}$ at $37^{\circ} \mathrm{C}$. Human ACE2-Fc or other SARS-CoV-2 RBD specific antibodies were used as a positive control to validate data collection in different panels of screening. Medium containing equal amount of pseudoviruses but no antibodies was used as blank control. The culture medium of ACE2293T cells was removed and then replaced by the antibody-pseudovirus mixture. All operations were conducted in the BSL-2 lab in Shanghai Jiao Tong University. After an additional $48 \mathrm{~h}$ incubation, the luminescence of each well was measured using a ONEGlo $^{\text {TM }}$ Luciferase Assay System (Promega) in the Infinite M200 Pro NanoQuant (TECAN). The acquired luminescence units were normalized to those of blank control wells. Dosedependent neutralization curves were fitted using a four-parameter nonlinear regression in GraphPad Prism Version 9.0.0.

Plaque reduction neutralization

Plaque reduction neutralization test was performed using SARS-CoV-2 WA1/2020 (US_WA-1/2020 isolate), Alpha- (B.1.1.7/UK, Strain: SARS-CoV2/human/USA/CA_CDC_5574/2020), Beta-(B.1.351/SA, Strain: hCoV-19/USA/MDHP01542/2021), Gamma- (P.1/Brazil, Strain: SARS-CoV-2/human/USA/MD-MDH0841/2021), and Delta-variants (B.1.617.2/Indian, Strain: GNL-751, a recently isolated strain from Galveston County, Texas) at Galveston National Laboratory at University of Texas Medical Branch at Galveston, Texas. Briefly, antibodies were 3-fold serially diluted in MEM medium (Gibco) from $20 \mu \mathrm{g} / \mathrm{mL}$ for preparing the working solution. The dilutions were mixed with equal volume of $100 \mathrm{TCID}_{50}$ virus in two replicates and incubated at room temperature for $1 \mathrm{~h}$. The mixture was then added into a 96-well plate covered with Vero cells. Blank controls and virus infection controls were set up simultaneously. After incubation at $37^{\circ} \mathrm{C}, 5 \% \mathrm{CO}_{2}$ for 3 days, cytopathic effect (CPE) was observed under microscope and plaques were counted for efficacy evaluation. Wells with CPE changes are recorded as "+", otherwise recorded as "-". IC $\mathrm{C}_{50}$ values were calculated according to the following equation: $I_{50}=$ Antilog $(D-C \times(50-B) /(A-B))$. Where $A$ indicates the percentage of inhibition higher than $50 \%$, B indicates the percentage of inhibition less than $50 \%, C$ is $\log _{10}$ (dilution factor), $D$ is $\log _{10}$ (Sample concentration which the inhibition is less than $50 \%$.

\section{ACE2 transgenic mouse protection}

AC70 human ACE2 transgenic mice (Taconic Biosciences) were divided into control (100 $\mu \mathrm{L}$ PBS) and treatment $(20,6.7$, or $2.2 \mathrm{mg} / \mathrm{kg}$ of $2 \mathrm{G} 1,100 \mu \mathrm{L})$ groups, with 14 in each group. Animal studies were carried out at Galveston National Laboratory at University of Texas Medical Branch at Galveston, Texas, an AAALAC accredited (November 24, 2020) and PHS OLAW approved (February 26, 2021) high-containment National Laboratory, based on a protocol approved by the Institutional Animal Care and Use Committee at UTMB at Galveston. Mice were challenged with 100 LD $_{50}$ of SARS-CoV-2 (US_WA-1/2020 isolate), Beta-(B.1.351/SA, Strain: hCoV-19/USA/MD-HP01542/2021), or Delta-variants 
(B.1.617.2/Indian, Strain: GNL-751, a recently isolated strain from Galveston County, Texas), provided through World Reference Center for Emerging Viruses and Arboviruses (WRCEVA) were used in the study. The first dose of antibody 2G1 and PBS were given 4 $\mathrm{h}$ post infection; and the second and third were given 2 days and 4 days post infection. Mice were clinically observed at least once daily and scored based on a 1 to 4 grading system that describes the clinical wellbeing. In the standardized 1 to 4 grading system, score 1 is healthy; Score 2 is with ruffled fur and lethargic; Score 3 is with additional clinical sign such as hunched posture, orbital tightening, increased respiratory rate, and/or $>15 \%$ weight loss; Score 4 is showing dyspnea and/or cyanosis, reluctance to move when stimulated, or $\geq 20 \%$ weight loss that need immediate euthanasia. Four mice in each group were euthanized at 4 days post infection for assessing viral loads and histopathology of lung and brain. The remaining 10 mice were continue monitored for morbidity and mortality for up to 12 days post infection.

\section{Rhesus macaque protection}

Rhesus macaques at six to seven years old were purchased from Hubei Tianqin Biotechnology Co., Ltd. All animal procedures and operations were approved by the ethical committee of Wuhan Institute of Virology, Chinese Academy of Sciences. SARS-CoV-2 strain 2019-nCoV-WIV04 (GISAID number: EPI_ISI_402124) was isolated from the bronchoalveolar lavage fluid of a patient who was infected COVID-19 in Wuhan in December 2019. Rhesus macaques were randomly divided into control group, low-dose (10 mg/kg of $2 \mathrm{G} 1$ ) and high-dose (50 mg/kg of $2 \mathrm{G} 1$ ) groups with one male and one female in each. Animals were endotracheally infected with $4 \mathrm{~mL}$ of $1 \times 10^{5} \mathrm{TCID} 50$ virus. Antibody $2 \mathrm{G} 1$ and PBS were intravenously given $24 \mathrm{~h}$ after infection. Rhesus macaques were monitored for disease-related changes during the period. Body weight and temperature were measured every day, and throat swab and anal swab samples were collected for virus titrating. Animals were euthanized at $7 \mathrm{dpi}$ and tissue samples were collected for virus examining. Viral RNA was extracted using the QIAamp Viral RNA Mini Kit (Qiagen). A onestep real-time quantitative PCR was used to quantify the viral RNA according to the supplier's instructions (HiScript ${ }^{\circledR}$ II One Step qRT-PCR SYBR® Green Kit, Vazyme Biotech Co., Ltd) together with primers for the RBD gene (RBD-qF1: 5'-CAATGGTTAAGGCAGG3'; RBD-qR1: 5'-CTCAAGGTCTGGATCACG-3').

\section{Antibody-Dependent Cellular Phagocytosis (ADCP)} In ADCP experiment, CD14 ${ }^{+}$monocytes (Allcells) were cultured and differentiated for 7 days to obtain macrophage cells. Macrophages were labeled with violet dye (ThermoFisher), and Jurkat cells with stable SARS-CoV-2 S expression were labeled with CFSE dye (ThermoFisher). 75,000 Jurkat cells were added to macrophage cells in a 96well plate in the presence of $2 \mathrm{G} 1$ or the isotype control antibody. After incubating at $37^{\circ} \mathrm{C}$ for 30 mins, the macrophages were digested and fixed with $4 \%$ paraformaldehyde, and the proportion of double-positive cell populations was analyzed by flow cytometry.

Pharmacokinetic study and toxicity test

For the pharmacokinetic study, BALB/c mice were tail intravenously injected with $2 \mathrm{G} 1$ (15, 
30 , or $60 \mathrm{mg} / \mathrm{kg}$ ), or equivalent volume of PBS. Three males and three females were in each subset. Blood samples were collected $0.5 \mathrm{~h}, 6 \mathrm{~h}, 1 \mathrm{~d}, 2 \mathrm{~d}, 4 \mathrm{~d}, 7 \mathrm{~d}, 10 \mathrm{~d}, 15 \mathrm{~d}, 21 \mathrm{~d}$, and $28 \mathrm{~d}$ after injection. Serum 2G1 concentration was quantified using ELISA. Briefly, Mouse Anti-human IgG Lambda (SouthBiotech) at $2 \mu \mathrm{g} / \mathrm{mL}$ was coated in ELISA plates. Serum samples and antibody 2G1 control were added into the plates and incubated for 1 h. After washing, a Goat Anti-human Fc HRP (Sigma) was used as secondary antibody with 1:6000 dilutions. After the chromogenic reaction by the HRP substrate (Solarbio), the plates were read at $450 \mathrm{~nm}$.

Crlj:CD1(ICR) mice were randomly divided into control (PBS), $15 \mathrm{mg} / \mathrm{kg}, 30 \mathrm{mg} / \mathrm{kg}$, and 60 $\mathrm{mg} / \mathrm{kg}$ groups for testing the in vivo toxicity of $2 \mathrm{G1}$, with three males and three females each group. Body weight was tracked every two days. Blood samples were collected 14 days after administration and mice were subsequently euthanized for tissue damage detection. Blood indicators including white blood cell count, red blood cell count, hemoglobin, and platelets were measured in multiple automated hematology analyzer (Sysmex XT-2000iV). Pathological changes of hearts, livers, spleens, lungs and kidneys were examined by hematoxylin-eosin (HE) staining.

\section{Expression and purification of $S$ protein}

The prefusion S extracellular domain (1-1208 a.a) (Genbank ID: QHD43416.1) was cloned into the pCAG vector (Invitrogen) with six proline substitutions at residues 817, 892, 899, 942, 986 and $987^{39}$, a "GSAS" substitution (instead of "RRAR") at residues 682 to 685 and a C-terminal T4 fibritin trimerization motif followed by one Flag tag.

This recombinant $S$ protein was overexpressed using the HEK 293F mammalian cells (Invitrogen) at $37^{\circ} \mathrm{C}$ under $5 \% \mathrm{CO}_{2}$ in a Multitron-Pro shaker (Infors, $130 \mathrm{rpm}$ ). For secreted $S$ protein production, about $1.5 \mathrm{mg}$ of the plasmid was premixed with $3 \mathrm{mg}$ of polyethylenimines (PEls) (Polysciences) in $50 \mathrm{~mL}$ of fresh medium for 15 mins before adding to cell culture, and transiently transfected into the cells, when the cell density reached $2.0 \times 10^{6}$ cells $/ \mathrm{mL}$. Cells were removed by centrifugation at $4000 \times \mathrm{g}$ for 15 mins and cell culture supernatant was collected sixty hours after transfection. The secreted $S$ proteins were purified by anti-FLAG M2 affinity resin (Sigma Aldrich). After loading two times, the anti-FLAG M2 resin was washed with the wash buffer containing $25 \mathrm{mM}$ Tris ( $\mathrm{pH}$ 8.0), $150 \mathrm{mM} \mathrm{NaCl}$. The protein was eluted with the wash buffer plus $0.2 \mathrm{mg} / \mathrm{mL}$ flag peptide. The eluent was then concentrated and subjected to gel filtration chromatography (Superose 6 Increase 10/300 GL, GE Healthcare) in buffer containing $25 \mathrm{mM}$ Tris (pH 8.0), $150 \mathrm{mM} \mathrm{NaCl}$. The peak fractions were collected and concentrated to incubate with mAb. The purified $S$ protein was mixed with the $2 G 1$ at a molar ratio of about 1:5 for one hour, respectively. Then the mixture was subjected to gel filtration chromatography (Superose 6 Increase 10/300 GL, GE Healthcare) in buffer containing $25 \mathrm{mM}$ Tris (pH 8.0), $150 \mathrm{mM}$ $\mathrm{NaCl}$. The peak fractions were collected for EM analysis.

\section{Cryo-EM sample preparation, data collection and data processing}

The peak fractions of complex were concentrated to about $2.5 \mathrm{mg} / \mathrm{mL}$ and applied to the grids. Aliquots $(3.3 \mu \mathrm{L})$ of the $\mathrm{S} / 2 \mathrm{G} 1$ complex were placed on glow-discharged holey carbon grids (Quantifoil Au R1.2/1.3). The grids were blotted for $2.5 \mathrm{~s}$ or $3.0 \mathrm{~s}$ and flash- 
frozen in liquid ethane cooled by liquid nitrogen with Vitrobot (Mark IV, ThermoFisher). The prepared grids were transferred to a Titan Krios operating at $300 \mathrm{kV}$ equipped with Gatan K3 detector and GIF Quantum energy filter. Movie stacks were automatically collected using AutoEMation ${ }^{40}$, with a slit width of $20 \mathrm{eV}$ on the energy filter and a defocus range from $-1.2 \mu \mathrm{m}$ to $-2.2 \mu \mathrm{m}$ in super-resolution mode at a nominal magnification of $81,000 \times$. Each stack was exposed for $2.56 \mathrm{~s}$ with an exposure time of $0.08 \mathrm{~s}$ per frame, resulting in a total of 32 frames per stack. The total dose rate was approximately $50 \mathrm{e}^{-} / \AA^{2}$ for each stack. The stacks were motion corrected with MotionCor $2^{41}$ and binned 2-fold, resulting in a pixel size of $1.087 \AA /$ pixel. Meanwhile, dose weighting was performed ${ }^{42}$. The defocus values were estimated with $\mathrm{Gctf}^{43}$.

Particles for $S$ in complex with $2 \mathrm{G} 1$ were automatically picked using Relion 3.0.6 $6^{44-47}$ from manually selected micrographs. After 2D classification with Relion, good particles were selected and subject to two cycle of heterogeneous refinement without symmetry using cryoSPARC ${ }^{48}$. The good particles were selected and subjected to Non-uniform Refinement (beta) with $\mathrm{C} 1$ symmetry, resulting in the 3D reconstruction for the whole structures, which was further subject to 3D auto-refinement and post-processing with Relion. For interface between S protein of SARS-CoV-2 and $2 \mathrm{G} 1$, the dataset was subject to focused refinement with adapted mask on each RBD-2G1 sub-complex to improve the map quality. The dataset of similar RBD-2G1 sub-complexes were combined if possible and necessary. The re-extracted dataset was 3D classified with Relion focused on RBD-2G1 sub-complex. Then the good particles were selected and subject to focused refinement with Relion, resulting in the 3D reconstruction of better quality on RBD-2G1 sub-complex. The resolution was estimated with the gold-standard Fourier shell correlation 0.143 criterion $^{49}$ with high-resolution noise substitution ${ }^{50}$. Refer to Supplementary information, Fig. S6-7 and Table $\mathrm{S} 1$ for details of data collection and processing.

For model building of the complex of S of SARS-CoV-2 with 2G1, the atomic model of the $S$ in complex 4A8 (PDB ID: 7C2L) were used as templates, which were molecular dynamics flexible fitted ${ }^{51}$ into the whole cryo-EM map of the complex and the focused-refined cryoEM map of the RBD-2G1 sub-complex, respectively. A Chainsaw ${ }^{52}$ model of the $2 \mathrm{G} 1$ was first obtained using the $4 A 8$ as a template, which was further manually adjusted based on the focused-refined cryo-EM map of the RBD-2G1 sub-complex with $\operatorname{Coot}^{53}$. Each residue was manually checked with the chemical properties taken into consideration during model building. Several segments, whose corresponding densities were invisible, were not modeled. Structural refinement was performed in Phenix ${ }^{54}$ with secondary structure and geometry restraints to prevent overfitting. To monitor the potential overfitting, the model was refined against one of the two independent half maps from the gold-standard $3 \mathrm{D}$ refinement approach. Then, the refined model was tested against the other map. Statistics associated with data collection, 3D reconstruction and model building were summarized in Supplemental information, Supplementary information, Table S1.

\section{Binding to $S$ mutants on cell surface}

Plasmids encoding full length SARS-CoV-2 S (GenBank ID: QHD43416.1) with one or more mutation sites were carried into HEK-293T cells using lipofectamine 3000 (ThermoFisher) according to the manufacturer's instruction. After 48 hours, cells were 
disassociated from the plates using a Cell Dissociation Buffer (ThermoFisher) followed by washing with PBS. Antibody $2 \mathrm{G} 1$ at $10 \mu \mathrm{g} / \mathrm{ml}$ was added into cells for a $30 \mathrm{~min}$ incubation. Subsequently, cells were washed and incubated with Alexa Fluor 647 labeled Goat antiHuman IgG (ThermoFisher) for 30 mins. After final washing, signals were acquired in flow cytometer $(\mathrm{BD})$ and the binding ability to $S$ mutants were evaluated by mean fluorescent intensity (MFI).

\section{Acknowledgments}

Authors would like to acknowledge following organizations and individuals for their assistances in the preparation of the manuscript: Professors Chuan Qin and Jiangning Liu from Institute of Laboratory Animal Sciences, CAMS \& PUMC, China for their support in initial cell based assay on neutralizing activity of the antibody generated in our lab; Dr. Liang Zhang of Jecho Biopharmaceuticals Co., Ltd. for his input on potential clinical applications of the antibodies; Professor Buyong Ma of SJTU for discussion and analysis on the structural interaction between the virus and antibody; We thank the cryo EM facility and the High-Performance Computing Center of Westlake University for providing supports. This work was funded by the National Natural Science Foundation of China (81773621, 82073751 to J.Z. 32022037, 31971123, 31800139 to Q.Z.); the National Science and Technology Major Project "Key New Drug Creation and Manufacturing Program" of China (No.2019ZX09732001-019 to J.Z.); the Key R\&D Supporting Program (Special support for developing medicine for infectious diseases) from the Administration of Chinese and Singapore Tianjin Eco-city to Jecho Biopharmaceuticals Ltd. Co.; Zhejiang University special COVID-19 grant 2020XGZX099 and Shanghai Jiao Tong University "Crossing Medical and Engineering" grant 20X190020003 to JZ. This work was funded by the Leading Innovative and Entrepreneur Team Introduction Program of Hangzhou, and Special Research Program of Novel Coronavirus Pneumonia of Westlake University and Tencent Foundation. We would like to express our sincere gratitude towards the generous supports from Tencent Foundation and Westlake Education Foundation.

\section{Author Contributions}

$\mathrm{LH}, \mathrm{HM}$ designed and conducted experiments on antibody binding activities, antibody neutralizing experiments using pseudovirus system and drafted manuscript. $\mathrm{LH}, \mathrm{HT}, \mathrm{HZ}$, LW, YK, YY, HY, HuiC, JZhang, YL conducted experiments on molecular discovery from blood sample to antibodies and characterization. MW, JL, YYue designed and executed animal study on metabolic profile and toxicology. CKT, AD, KRK, BHP designed and executed in vitro and in vivo study on virus neutralizing activity. $\mathrm{XX}$ provided technical instructions on antibody screening from B cells. JG provided critical discussions and manuscript editing. YX, HJ coordinated project on molecular discovery, characterization, preparation, and provided critical discussions on in vitro and in vivo animal study on virus neutralization. $X Z, Z W, L Y, Y C h e n$ coordinated blood sample collection from convalescent individuals and facilitated B-cell screening. ZW, YH, YChang, GL, GcL, JJS, LLM, ZX conducted sample preparation, quality control, and product characterization. QZ conceived the project on structure analysis. YG designed and did the cryo-EM experiments. $Y Z$ solved the cryo-EM structures and $Y G$ and $Y Z$ analyzed the cryo-EM structures and made figures. 
$661 \mathrm{SW}, \mathrm{HH}, \mathrm{AW}, \mathrm{KY}, \mathrm{ZS}, \mathrm{HuaC}$, LiZ conducted experiments on antibody expression, analytical

662 development, and characterization. WX, SZ, TJ, conducted in vitro virus neutralizing

663 assays. YB, BZ coordinate project activities and provided critical discussion. JZhu

664 designed the overall project, organized and coordinated activities from all participating

665 institutes, and revised manuscript.

666

667

\section{Conflict of Interest}

668

We declare that none of the authors have competing financial interests.

669

670

671

672

673

674

675

676

677

678

679

680

681

682

683

684

685

686

687

688

689

690

691

692

693

694 

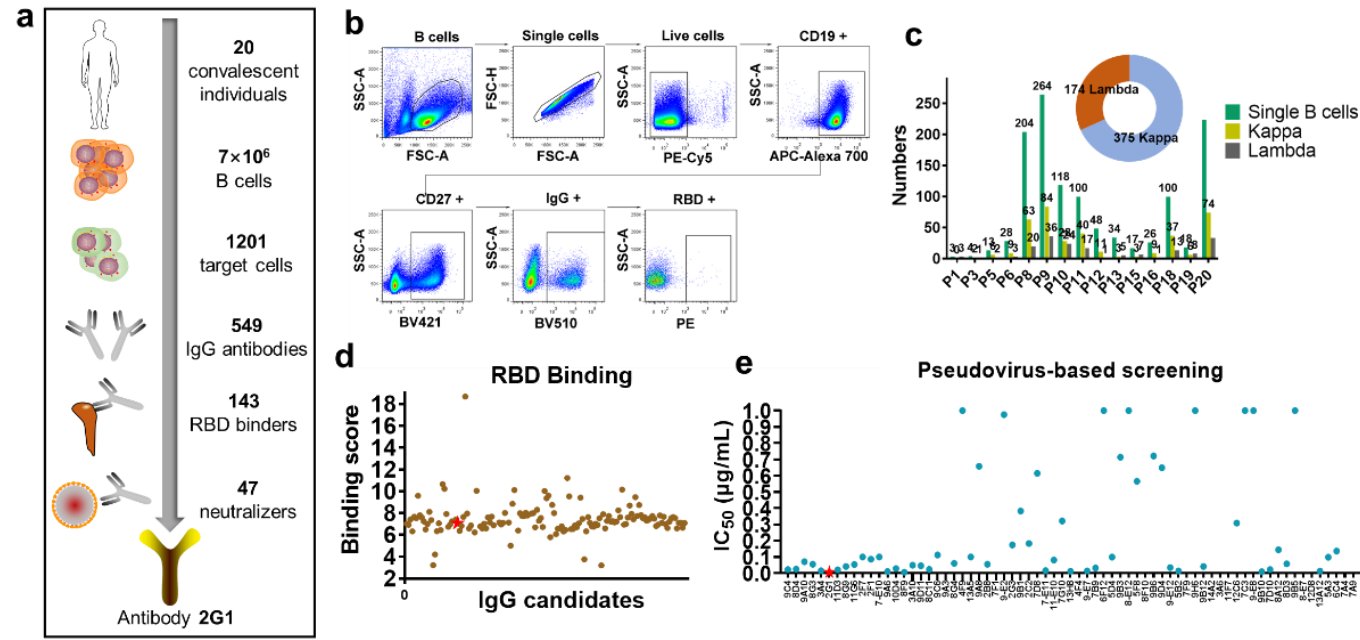

Fig. 1 Cell isolation, antibody cloning, and candidate panning. a, Isolation strategy of highly potent neutralizing antibodies as depicted by a diagram. b, RBD-specific B cells were isolated from convalescent subjects of SARS-CoV-2 infection by fluorescenceactivated cell sorting. The $7 \mathrm{ADD}^{-} / \mathrm{CD} 19^{+} / \mathrm{CD}^{2} 7^{+} / \mathrm{lgG}{ }^{+} / \mathrm{RBD}^{+}$gate is shown and highlighted in the boxes. c, Statistics of the number of paired antibodies from each subject, as well as the number of kappa and lambda subtypes. $\mathbf{d}$, Binding scores of antibody candidates against SARS-CoV-2 RBD as measured by ELISA and scores higher than 2 are presented. $2 \mathrm{G} 1$ is highlighted in red. e, Candidate panning using a WA1/2020 pseudovirus-based screening model. Antibodies were 10 -fold serially diluted from $10^{1} \mu \mathrm{g} / \mathrm{mL}$ to $10^{-4} \mu \mathrm{g} / \mathrm{mL}$. 
a

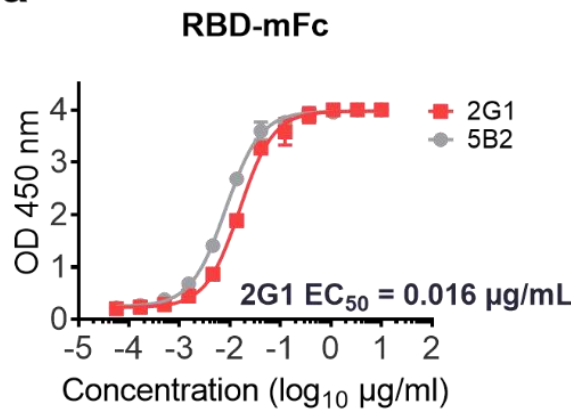

b

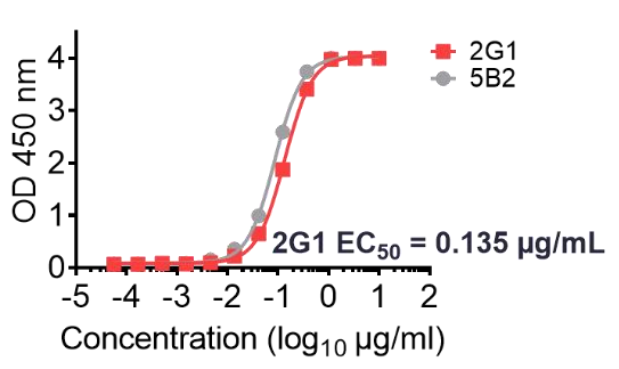

C WA1/2020 pseudovirus

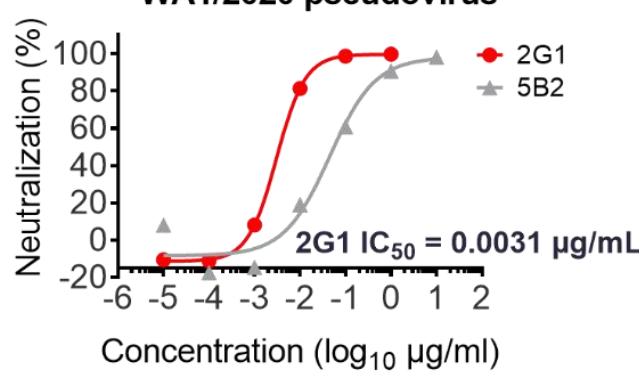

\section{d}

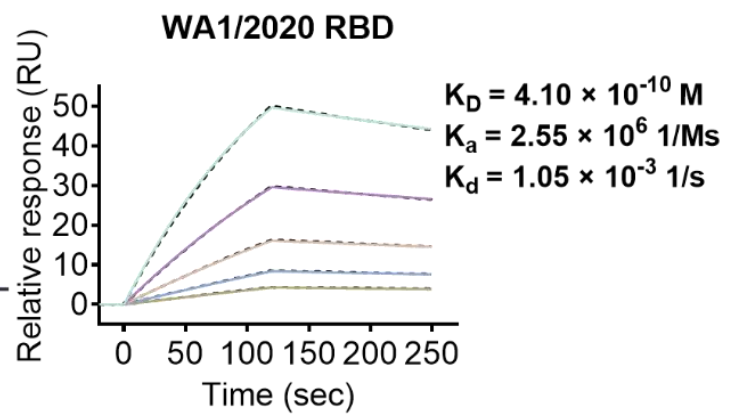

Fig. 2 Characterization of 2G1 using WA1/2020 related S and RBD proteins and pseudovirus. a-b, $2 \mathrm{G} 1$ concentration-dependently binds to RBD-mFc (a) and $\mathrm{S}$ trimer (b) of SARS-CoV-2 in ELISA test. A neutralizing antibody 5B2 targeting SARS-CoV-2 RBD was used as control. Values from two replicates are shown as mean \pm S.D. c, Serial tenfold-diluted 2G1 was incubated with SARS-CoV-2 WA1/2020 pseudovirus and used to infect 293T-ACE2 cells. After a $48 \mathrm{~h}$ incubation, the infection was quantified using a fluorescence detection kit. d, Binding kinetics of $2 \mathrm{G} 1$ to SARS-CoV-2 RBD in SPR. Serial dilutions of $2 \mathrm{G} 1 \mathrm{Fab}$ were flowed through a chip fixed with RBD recombinant protein. The kinetics data were fitted with results from different concentrations. 


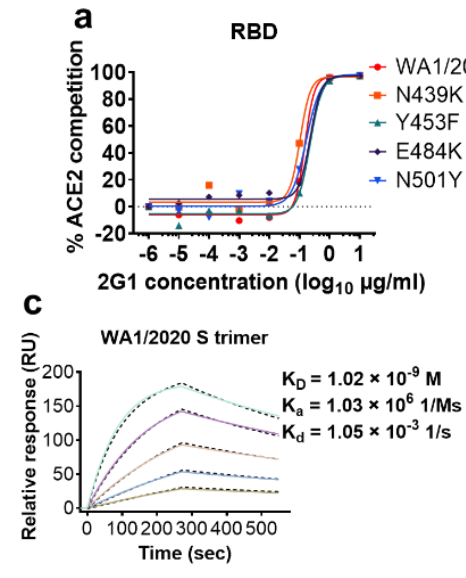

$\mathrm{IC}_{50}(\mu \mathrm{g} / \mathrm{mL})$

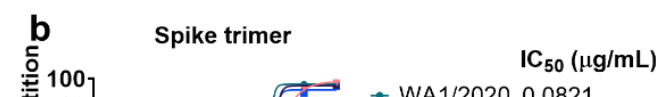

0.1504

0.1050

$-\mathrm{Y} 453 \mathrm{~F} \quad 0.2225$

- E484K 0.1951

0.1672
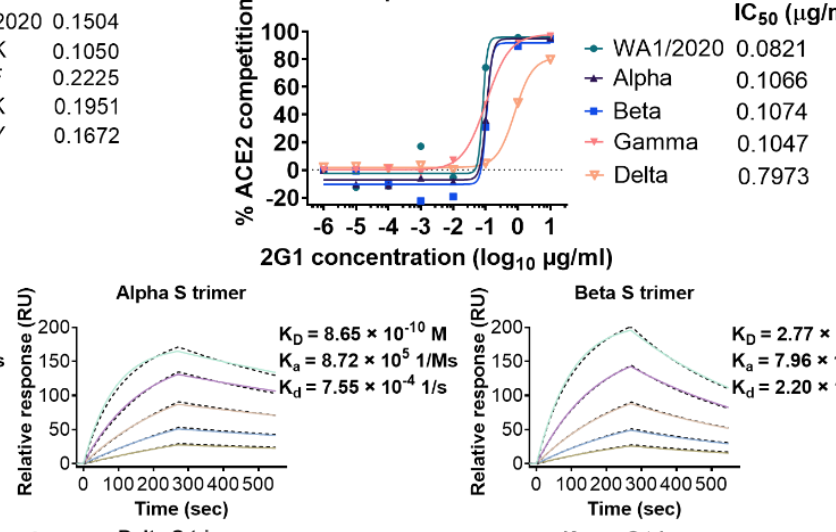
$2 \mathrm{G} 1$ concentration $\left(\log _{10} \mu \mathrm{g} / \mathrm{ml}\right)$
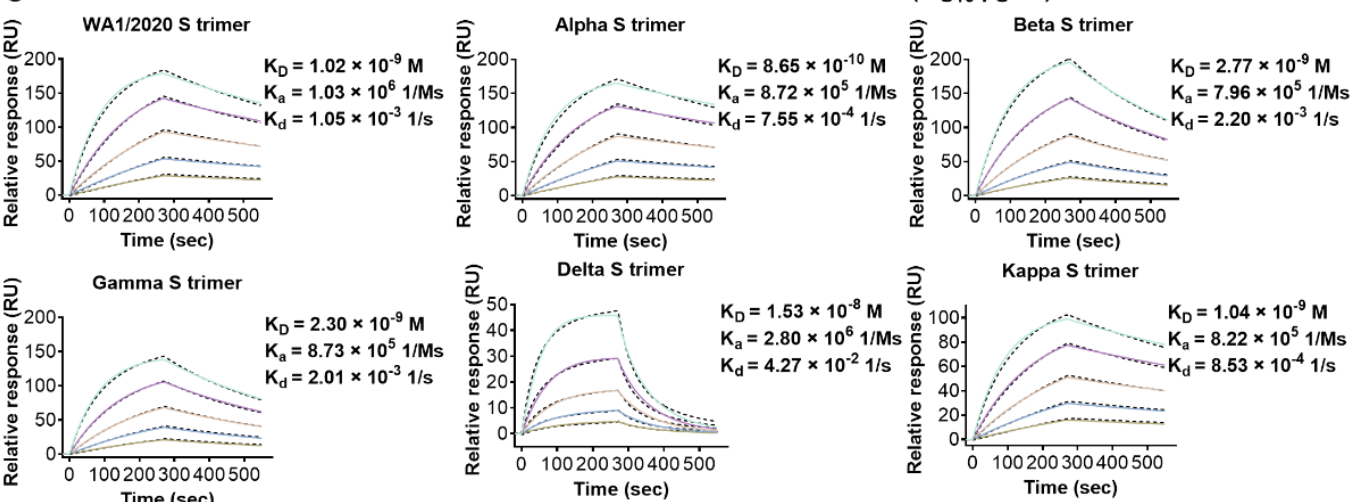

appa S

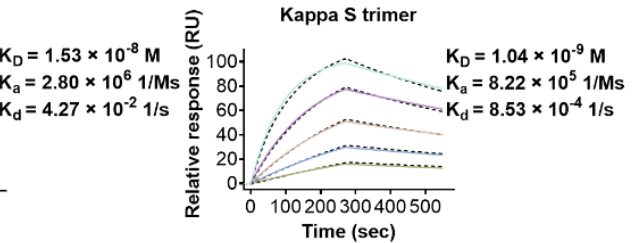

Fig. 3 Binding and blocking characteristics of 2G1 to SARS-CoV-2 variants. a-b, 2G1 competitively blocked the ACE2 binding to single point mutant RBD proteins (a) and VOC S trimers (b). c, Affinity analysis of $2 \mathrm{G} 1$ bound to $S$ trimers of SARS-CoV-2 WA1/2020, Alpha, Beta, Gamma, Kappa and Delta by SPR. Chips fixed with S trimers were loaded on a BIAcore $8 \mathrm{~K}$ system. $2 \mathrm{G} 1 \mathrm{Fab}$ varied from $1.250 \mu \mathrm{g} / \mathrm{mL}$ to $0.039 \mu \mathrm{g} / \mathrm{mL}$ were injected over the chips for measuring the real-time association and dissociation parameters. 
b
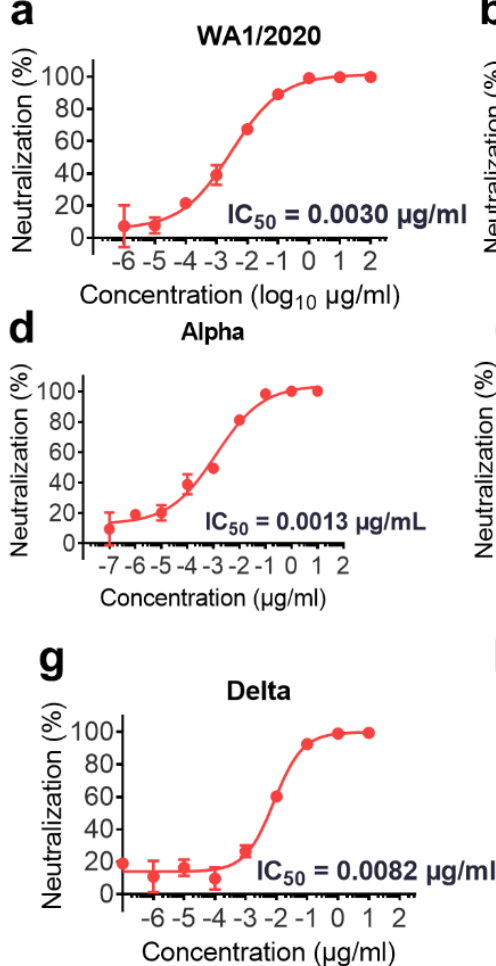

b $\quad$ 6614G

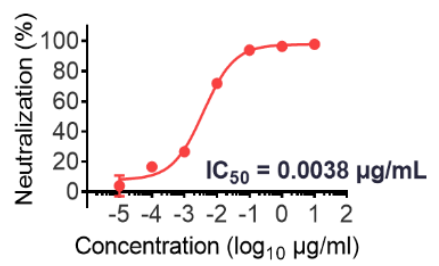

e

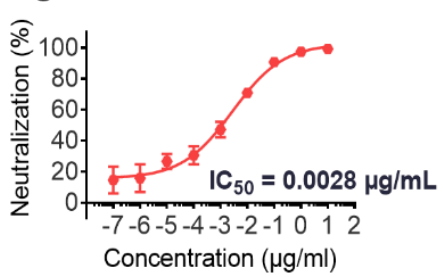

h

\begin{tabular}{ccc}
\hline \multicolumn{2}{|c}{ Live virus neutralization $\mathrm{IC}_{50}(\mu \mathrm{g} / \mathrm{ml})$} & $\mathrm{IC}_{100}(\mu \mathrm{g} / \mathrm{ml})$ \\
\hline WA1/2020 & 0.0240 & 0.0411 \\
Alpha & 0.0138 & 0.0411 \\
Beta & 0.0046 & 0.0137 \\
Gamma & 0.0079 & 0.0137 \\
Delta & 0.0240 & 0.0411 \\
\hline
\end{tabular}
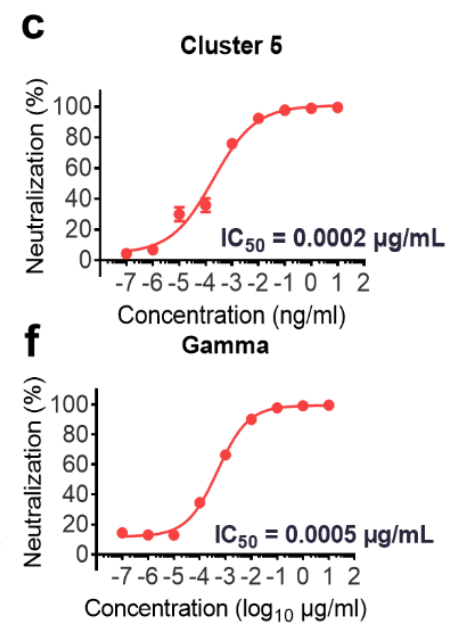

Fig. 4 Extensive neutralization of $2 \mathrm{G} 1$ against SARS-CoV-2 variants. a-g, Neutralization of $2 \mathrm{G} 1$ to diverse SARS-CoV-2 pseudoviruses. Pseudoviruses with active titer higher than $1 \times 10^{7} \mathrm{TU} / \mathrm{mL}$ were employed in this study. Concentration-dependent neutralization of $2 \mathrm{G} 1$ was quantified by detecting the fluorescence from the luciferase reporter. Data in duplicate are displayed as mean \pm S.D. $\mathbf{h}$, Live virus neutralization by 2G1. 100 TCID50 of SARS-CoV-2 (WA1/2020, Alpha, Beta, gamma and Delta) were incubated with threefold-diluted 2G1 and then added to Vero E6 cells. After a 3-day incubation, cytopathic effect (CPE) was assessed by counting the plaque formation. 

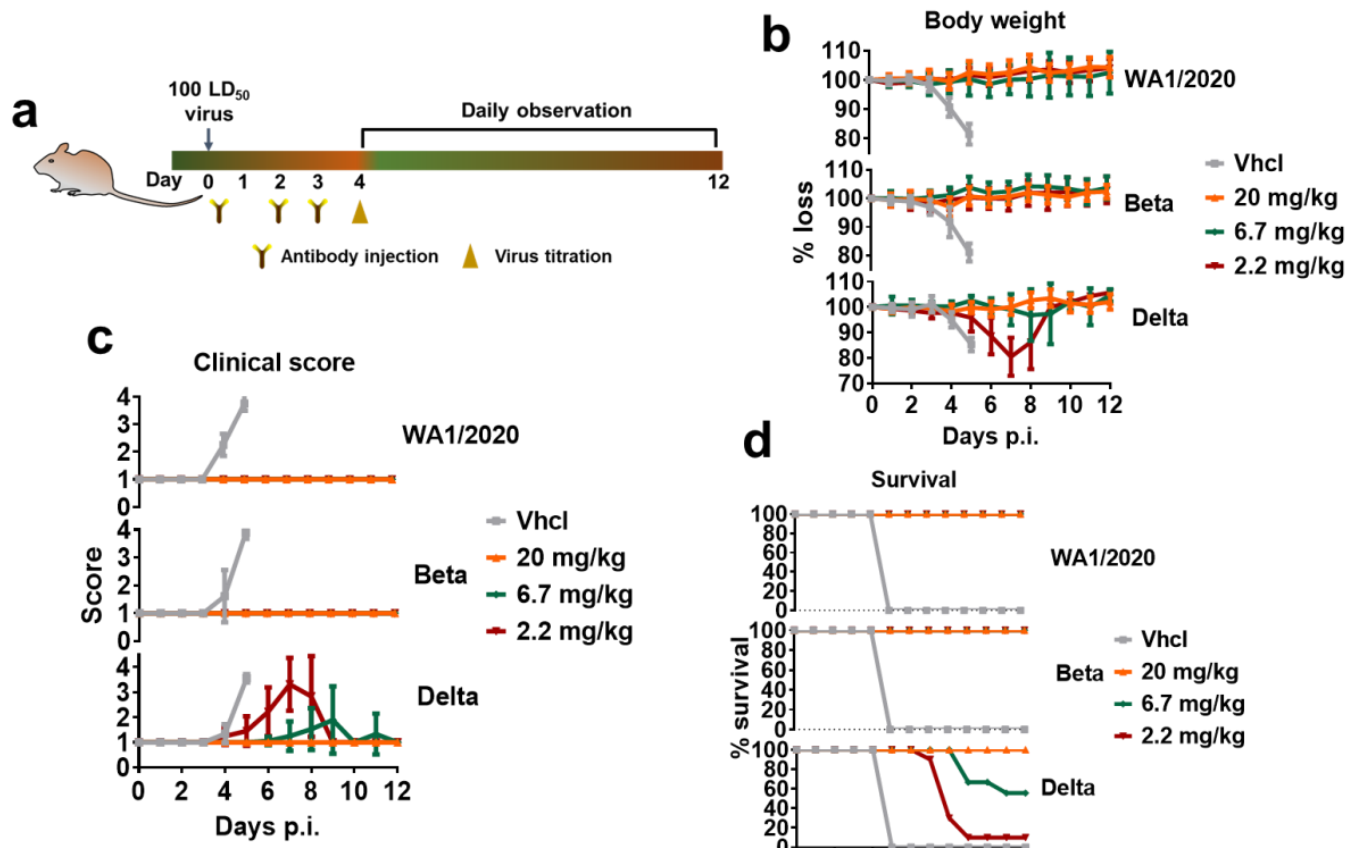

Fig. 5 Therapeutic efficacy of $2 \mathrm{G} 1$ against SARS-CoV-2 variants in transgenic mice. a, High permissive AC70 human ACE2 transgenic mice were challenged with $100 \mathrm{LD}_{50}$ of SARS-CoV-2 WA1/2020, Beta- or Delta- variants, followed by $20,6.7$, or $2.2 \mathrm{mg} / \mathrm{kg}$ of $2 \mathrm{G} 1$ treatment $(n=14)$. A 12-day clinical observation was implemented. $\mathbf{b}$, Body weight change of mice. c, Clinical illness of mice was assessed based on a standardized 1 to 4 grading system that describes the clinical wellbeing of mice. $\mathbf{d}$, Mortality of mice. Data are shown as mean \pm S.D. Vhcl, vehicle control; p.i., post infection. 
a

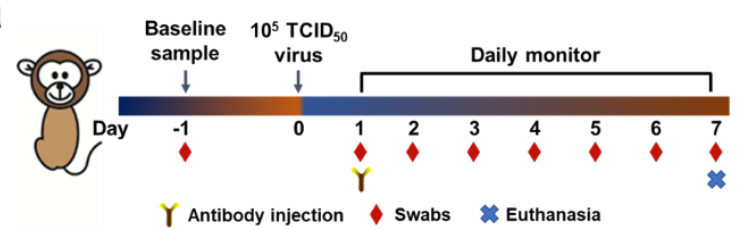

C

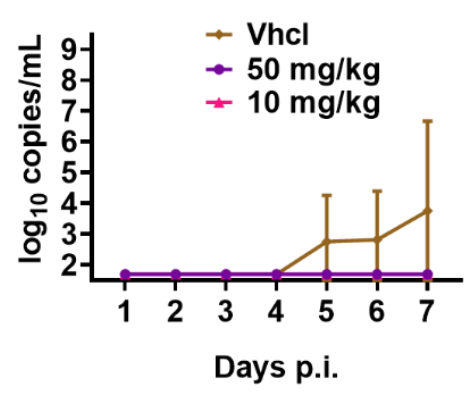

b

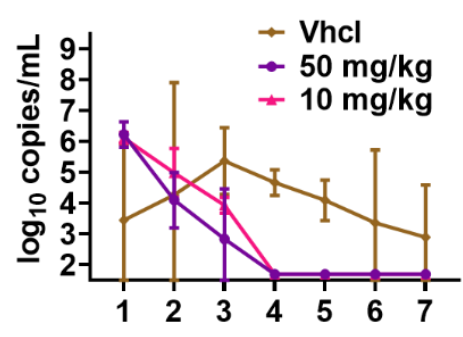

Days p.i.

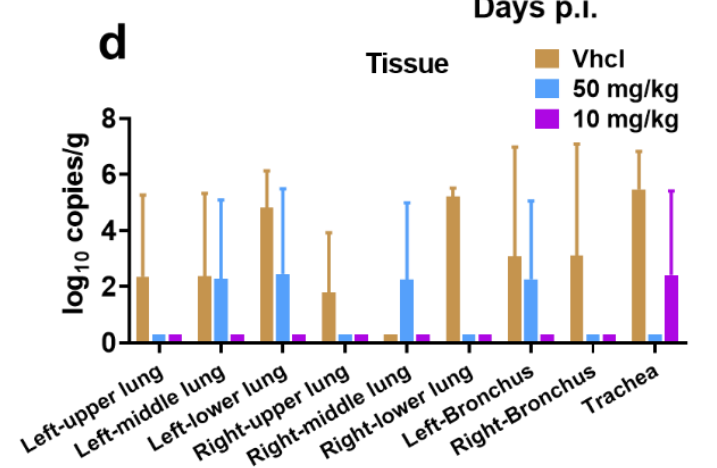

777

Fig. 6 Therapeutic efficacy of 2G1 against SARS-CoV-2 variants in rhesus macaques. a, One male and one female rhesus macaques in each group were endotracheally challenged with $1 \times 10^{5}$ TCID50 of SARS-CoV-2. $2 \mathrm{G} 1$ at $10 \mathrm{mg} / \mathrm{kg}$ or $50 \mathrm{mg} / \mathrm{kg}$, or equal amount of PBS were intravenously given at $1 \mathrm{dpi}$. Throat and anal swabs were sampled daily until 7 dpi. b, Viral load in throat swab. c, Viral load in anal swab. d, Viral load in lungs, tracheas, and bronchi. Data with duplications are shown as mean \pm S.D. p.i., post infection. 


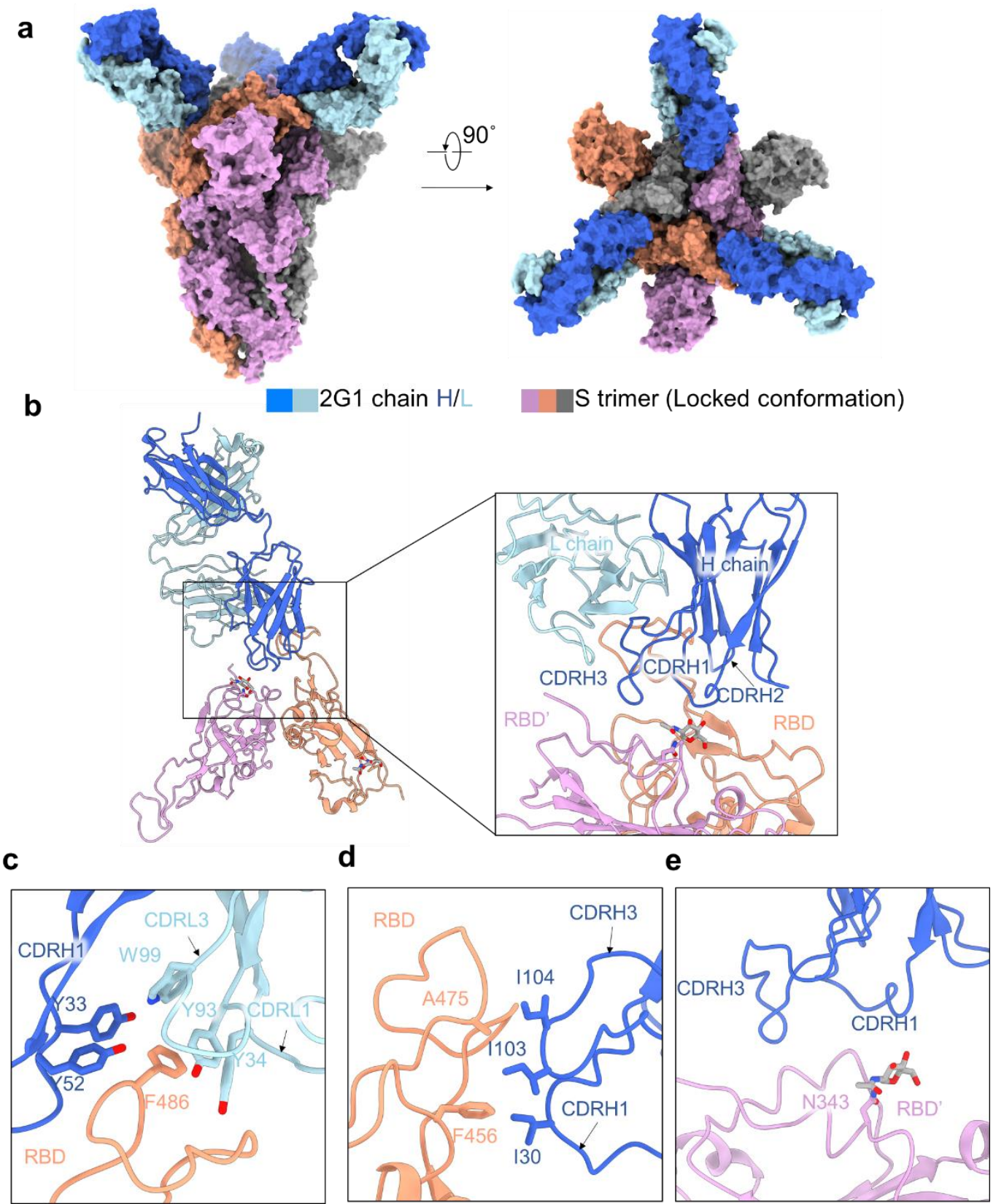

Fig. 7 Cryo-EM structure of 2G1 and the complex with WA1/2020 S protein. a, The domain-colored cryo-EM map of SARS-CoV-2 S ectodomain trimer and 2G1 Fab fragments complex is shown, viewed along two perpendicular orientations. The heavy and light chains of $2 \mathrm{G} 1$ are colored blue and cyan, respectively. $\mathbf{b}$, The three protomer of trimeric $S$ protein are colored grey, orange and pink. c-e, The binding interface between $2 \mathrm{G} 1$ and RBD and adjacent RBD'. RBD and $2 \mathrm{G1}$ interact each other mainly through hydrophobic interactions (c and d). 2G1 heavy chain (CDRH3 and CDRH1) lie above the adjacent RBD' (e). 
a

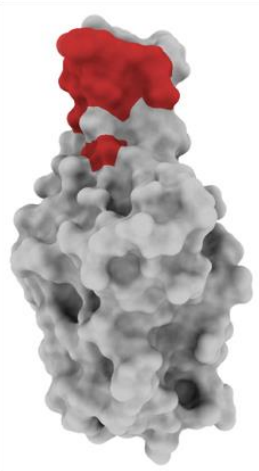

S2E12

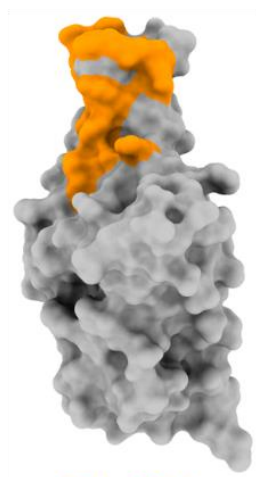

B1-182.1

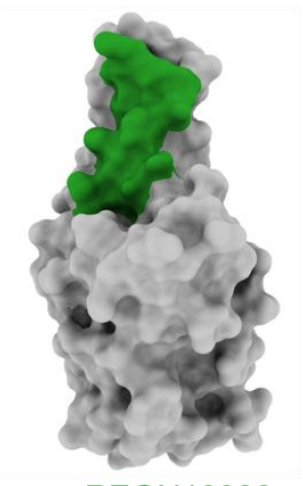

REGN10933

b
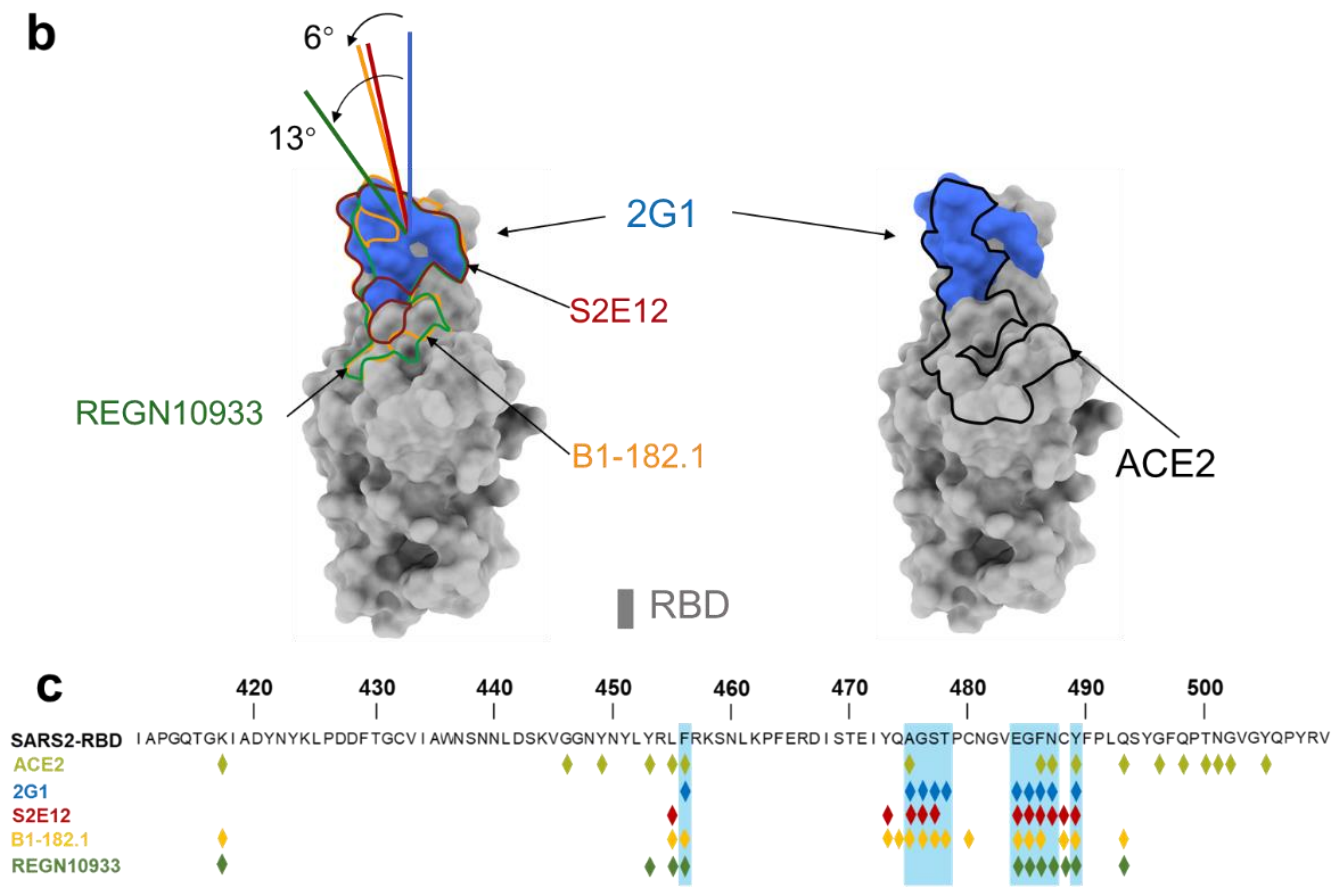

Fig. 8 Analysis of different binding modes of 2G1, S2E12, B1-182.1 and REGN10933. a, The epitope surfaces of S2E12, B1-182.1 and REGN10933 on S protein are in red, orange and green, respectively. b, Comparison of binding modes of 2G1, S2E12, B1-182.1 and REGN10933. The epitope surface of 2G1 is in blue. The borderlines of ACE2-binding site, S2E12, B1-182.1 and REGN10933 are shown in black, red, orange and green respectively. The connecting lines between the center of $2 \mathrm{G} 1 \mathrm{Fab}$ and RBD is taken as the principal axis, and axis of Fab S2E12, B1-182.1 are rotated $6^{\circ}$ and REGN10933 is rotated $13^{\circ}$ approximately. c, Mapping of S2E12, B1-182.1 and REGN10933 epitopes on RBD. 
807

808

809

810

811

812

813

814

815

816

817

818

819

820

821

822

823

824

825

826

827

828

829

830

831

832

833

834

835

836

837
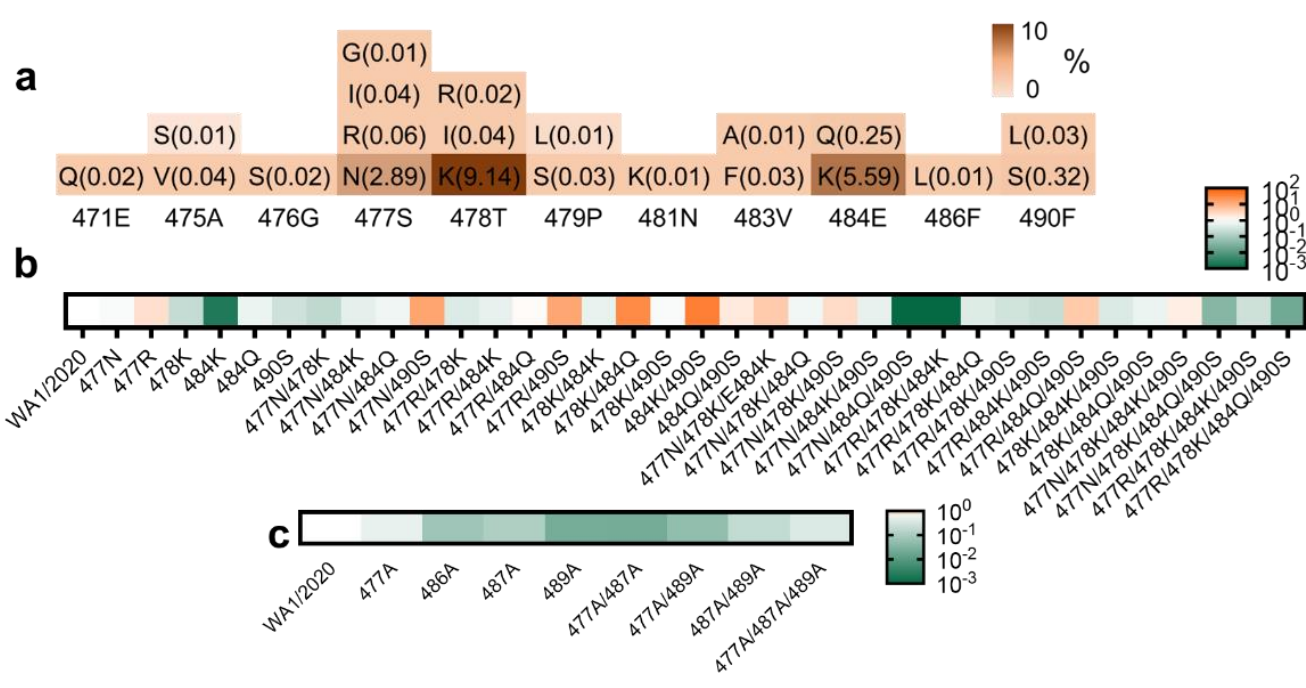

Fig. 9 Identification of critical binding residues for 2G1. a, Statistics of mutation proportion in RBD residue 471Glu - 490Phe where key for $2 \mathrm{G} 1$ epitope from GISAID database as of August 2021. b, Identification of critical binding residues for 2G1. Spike genes with high frequency mutation sites between 471Glu and 490Phe $(>0.05 \%)$ were cloned and transiently expressed on the surface of 293T cells. The binding ability of 2G1 to these mutant $S$ proteins was measured by flow cytometry. The fold change of binding ability was normalized by comparing to WA1/2020 S protein. c, Mutations in the key interaction sites of $2 \mathrm{G} 1$ that affects the binding ability of $2 \mathrm{G} 1$ to varying degrees. 
a

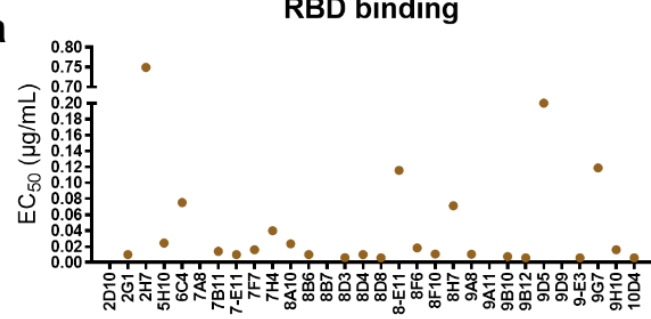

b

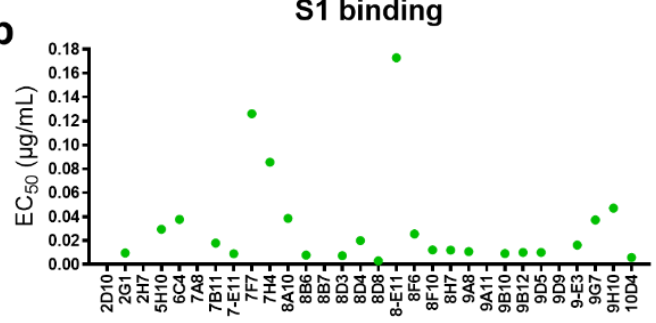

Fig. S1 Evaluation of binding and neutralization of selected antibody candidates. ab, Candidates' $\mathrm{EC}_{50}$ in the concentration-dependent RBD (a) and S1 (b) binding test using ELISA. Antigens were 3-fold serially diluted from $0.300 \mu \mathrm{g} / \mathrm{mL}$ to $0.0012 \mu \mathrm{g} / \mathrm{mL}$.
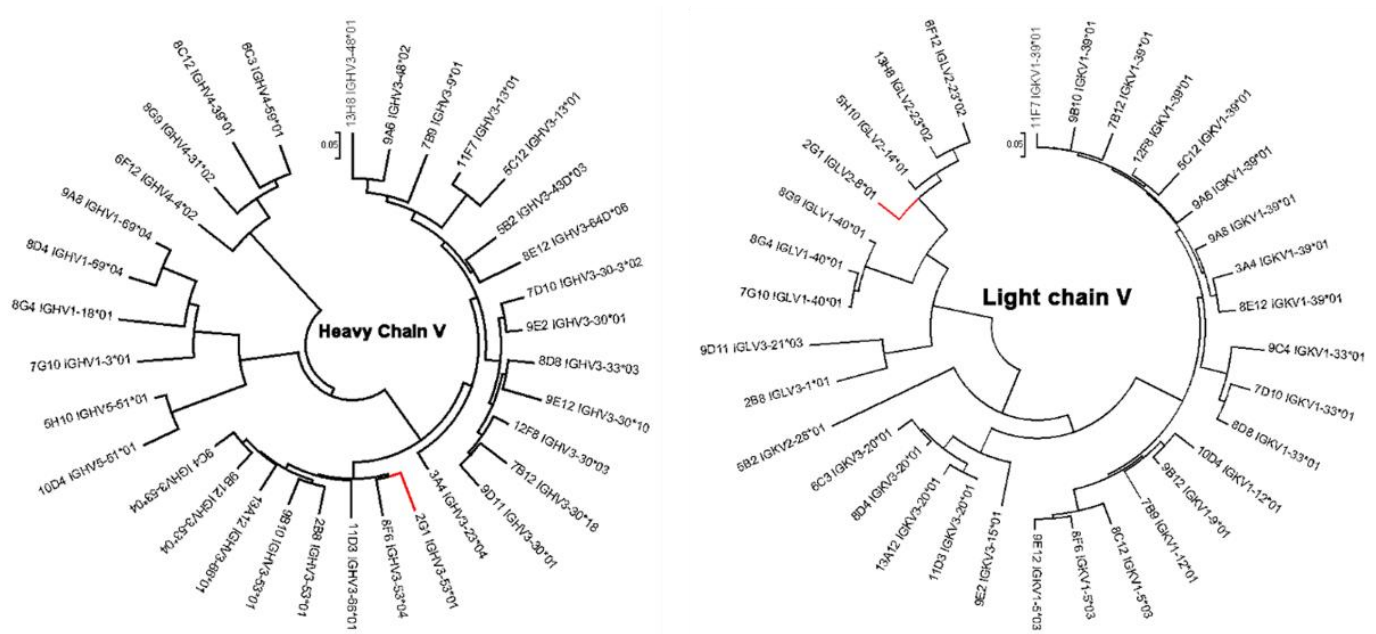

Fig. S2 Germline identification of VH and VL. Germline gene distribution of the heavy chain and light chain of 33 candidates and their clustering analysis. 


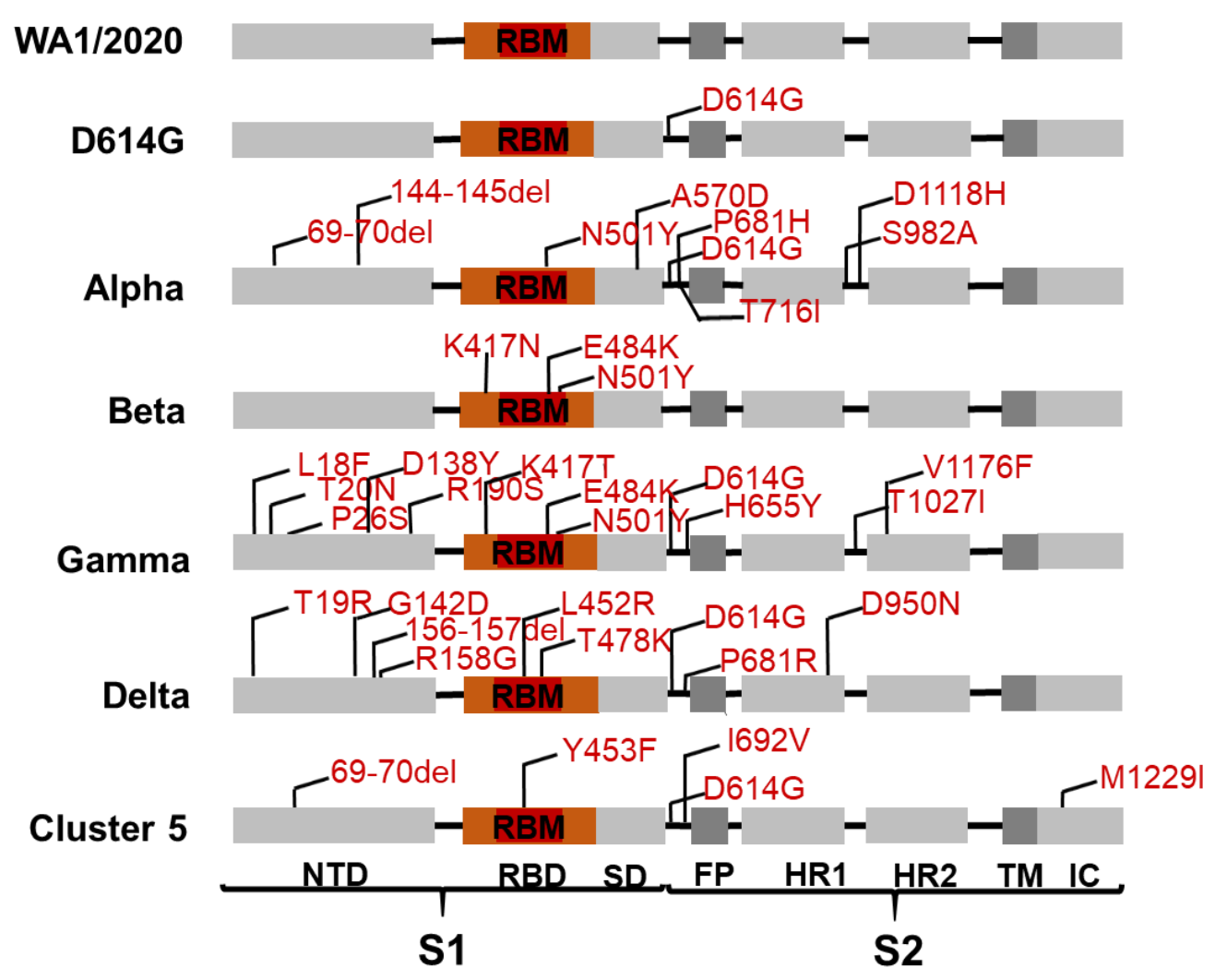

Fig. S3 Mutational sites of pseudoviruses used in this report. The spike region of SARS-CoV-2 is displayed in different modules. The mutation sites are annotated in corresponding positions in detail. RBD is highlighted in saffron yellow and RBM is highlighted in red. NTD, N-terminal domain; RBD, receptor binding domain; RBM, receptor binding motif; SD, subdomain; FP, fusion peptide; HR1, heptad repeats 1; HR2, heptad repeats 2; TM, transmembrane region; IC, intracellular region. 

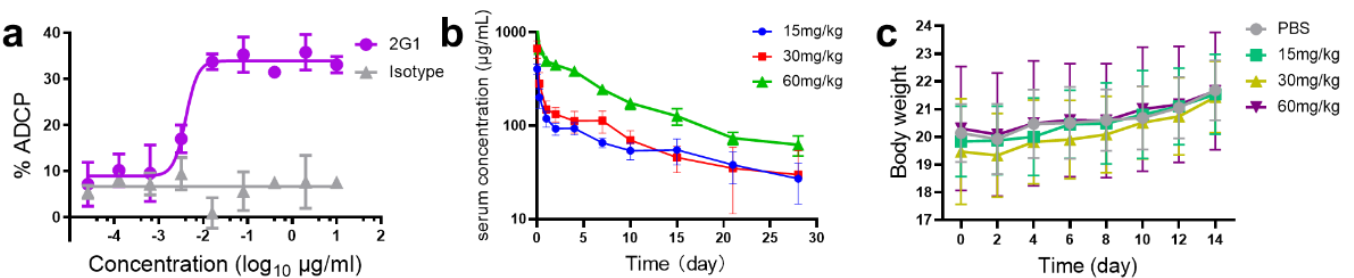

me (day)
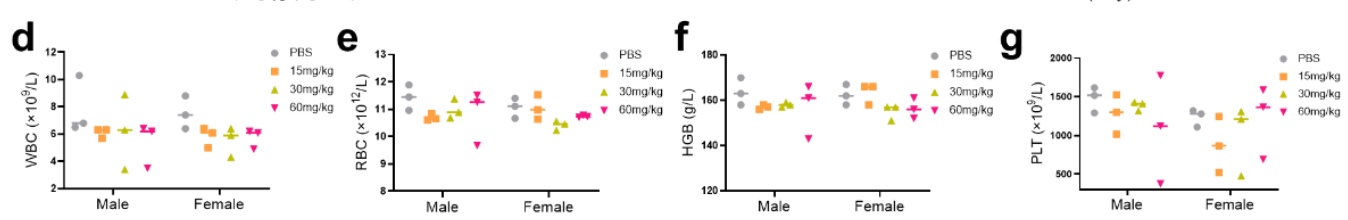

Fig. S4 2G1 induces cellular phagocytosis but no evident adverse effects. a, Antibody-dependent cellular phagocytosis (ADCP) induced by $2 \mathrm{G} 1$. Jurkat cells with stable $S$ expression were incubated with macrophages in the presence of different concentrations of $2 \mathrm{G} 1$. After incubating at $37^{\circ} \mathrm{C}$ for 30 mins, the proportion of Jurkat cells phagocytosed by macrophages was detected by flow cytometry. b, Pharmacokinetic study of $2 \mathrm{G} 1$. $B A L B / c$ mice were treated with different doses of $2 \mathrm{G} 1$, and blood samples were collected at different time points. The serum concentration of $2 \mathrm{G} 1$ was measured by ELISA. c-g, Adverse effect study of 2G1. Crlj:CD1(ICR) mice were treated with different doses of $2 \mathrm{G} 1$. Body weight of mice was tracked (c). The blood routine indexes including WBC (d), RBC (e), HGB (f), and PLT (g) were measured 14 days later. WBC, white blood cell count; RBC, red blood cell count; HGB, hemoglobin; PLT, platelets. Data are presented as mean \pm S.D.
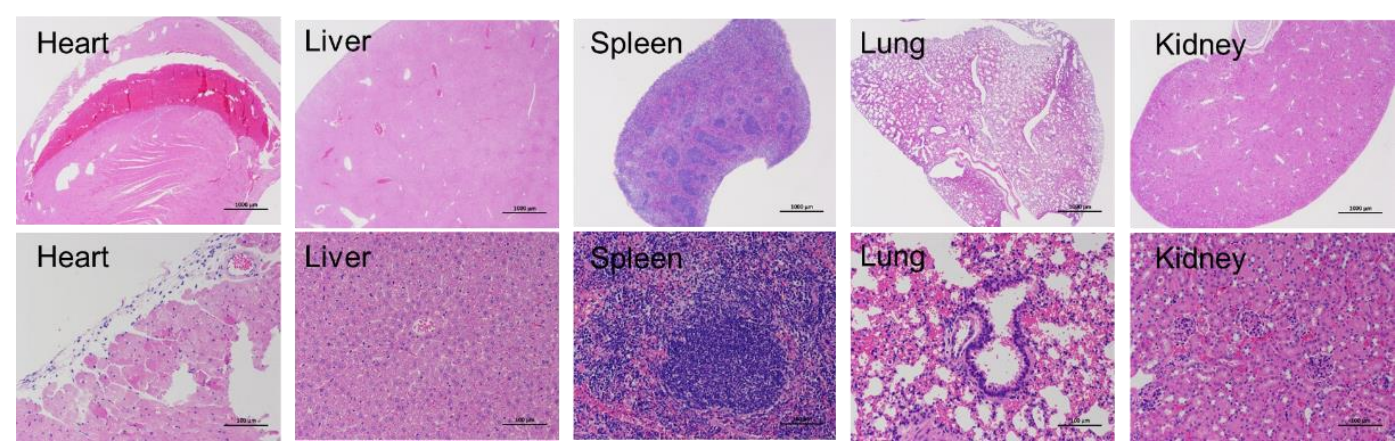

Fig. S5 Organ toxicity study. Crlj:CD1(ICR) mice were treated with 15, 30, or $60 \mathrm{mg} / \mathrm{kg}$ of 2G1. Inflammatory damage of hearts, livers, spleens, lungs and kidneys were checked by hematoxylin-eosin (HE) staining. No apparent pathological changes were observed. Representative sections from $60 \mathrm{mg} / \mathrm{kg}$ group are displayed. 
a
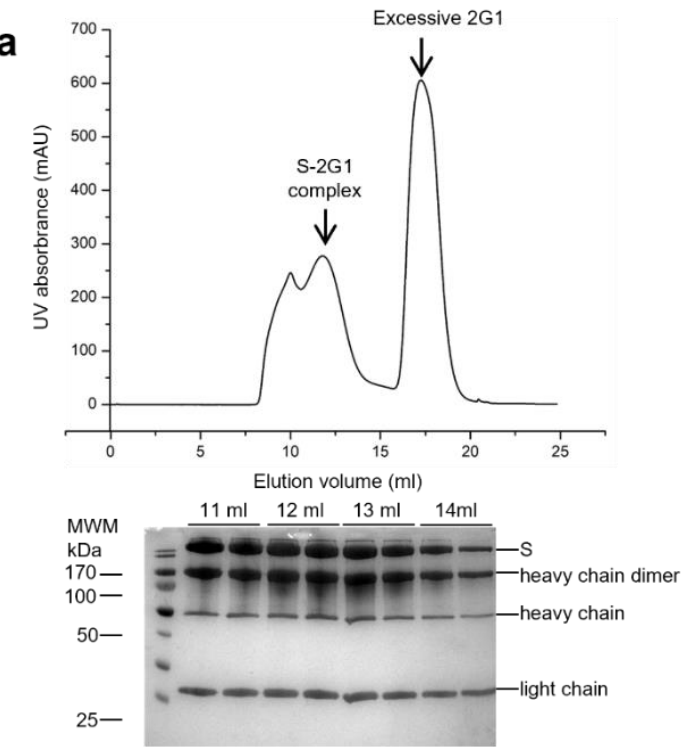

C

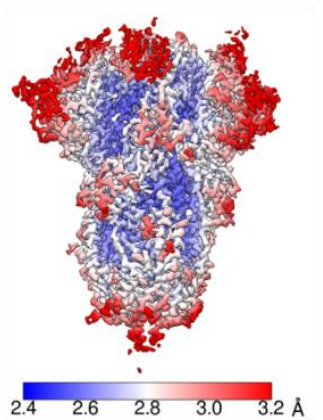

f
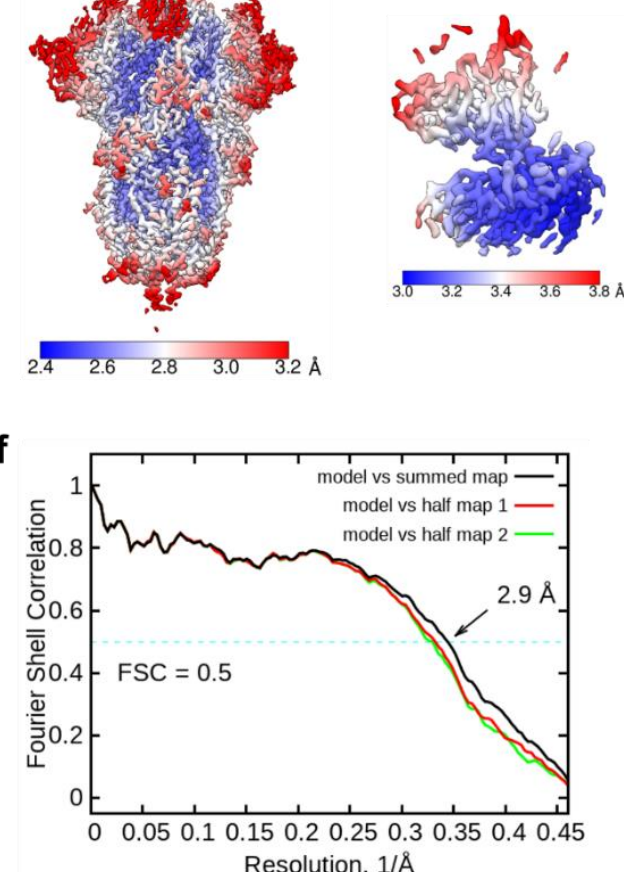

g b

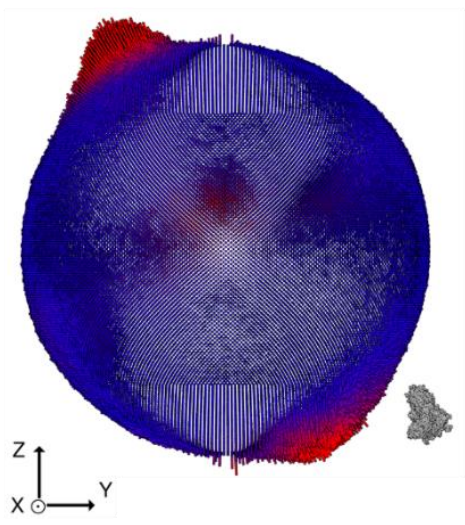

e
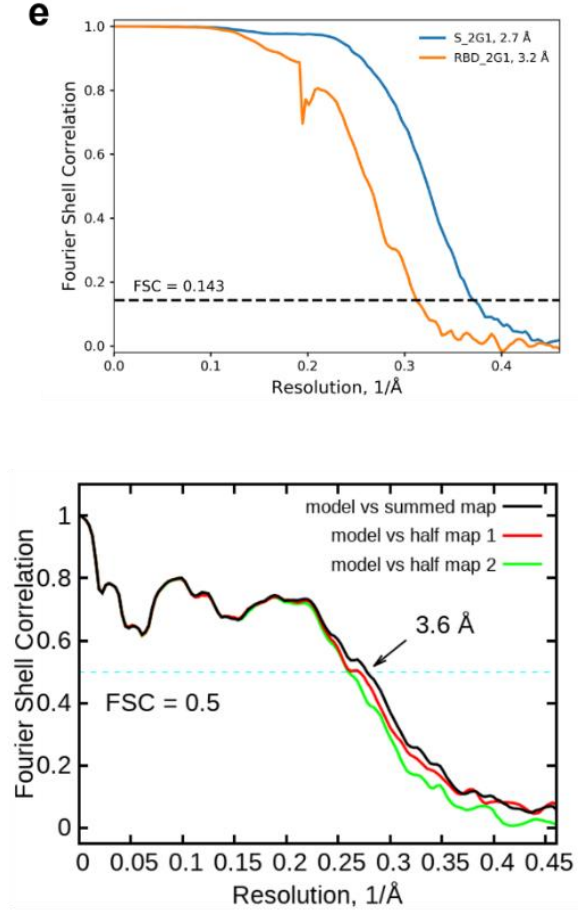

Fig. S6 Cryo-EM analysis of SARS-CoV-2 $S$ trimer in complex with 2G1. a, Representative gel filtration chromatography purification profile of the SARS-CoV-2 S extracellular domain in complex with $2 \mathrm{G} 1$. b. Euler angle distribution in the final $3 D$ reconstruction of $S$ bound with $2 \mathrm{G} 1$. c-d, Local resolution map for the 3D reconstruction of overall structure and RBD-2G1 sub-complex, respectively. e, FSC curve of the overall structure (blue) and RBD-2G1 sub-complex (orange). $\mathbf{f}, \mathrm{FSC}$ curve of the refined model of $S$ bound with 2G1 versus the overall structure that it is refined against (black); of the model refined against the first half map versus the same map (red); and of the model refined against the first half map versus the second half map (green). The small difference between the red and green curves indicates that the refinement of the atomic coordinates did not suffer from overfitting. g, FSC curve of the refined model of RBD-2G1 sub-complex, which is same to the $f$. 
a

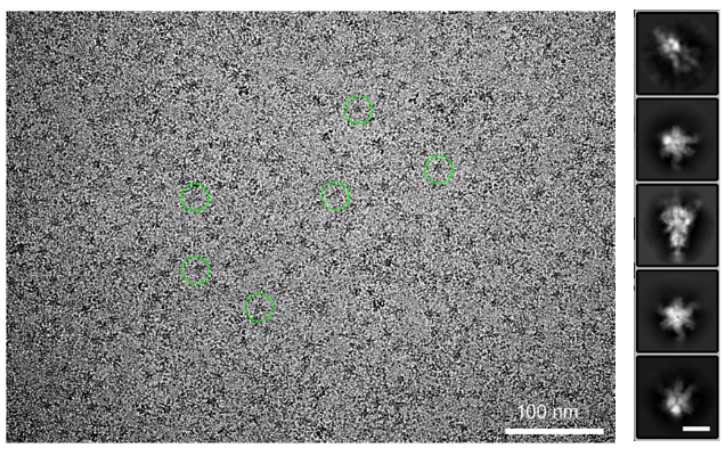

b

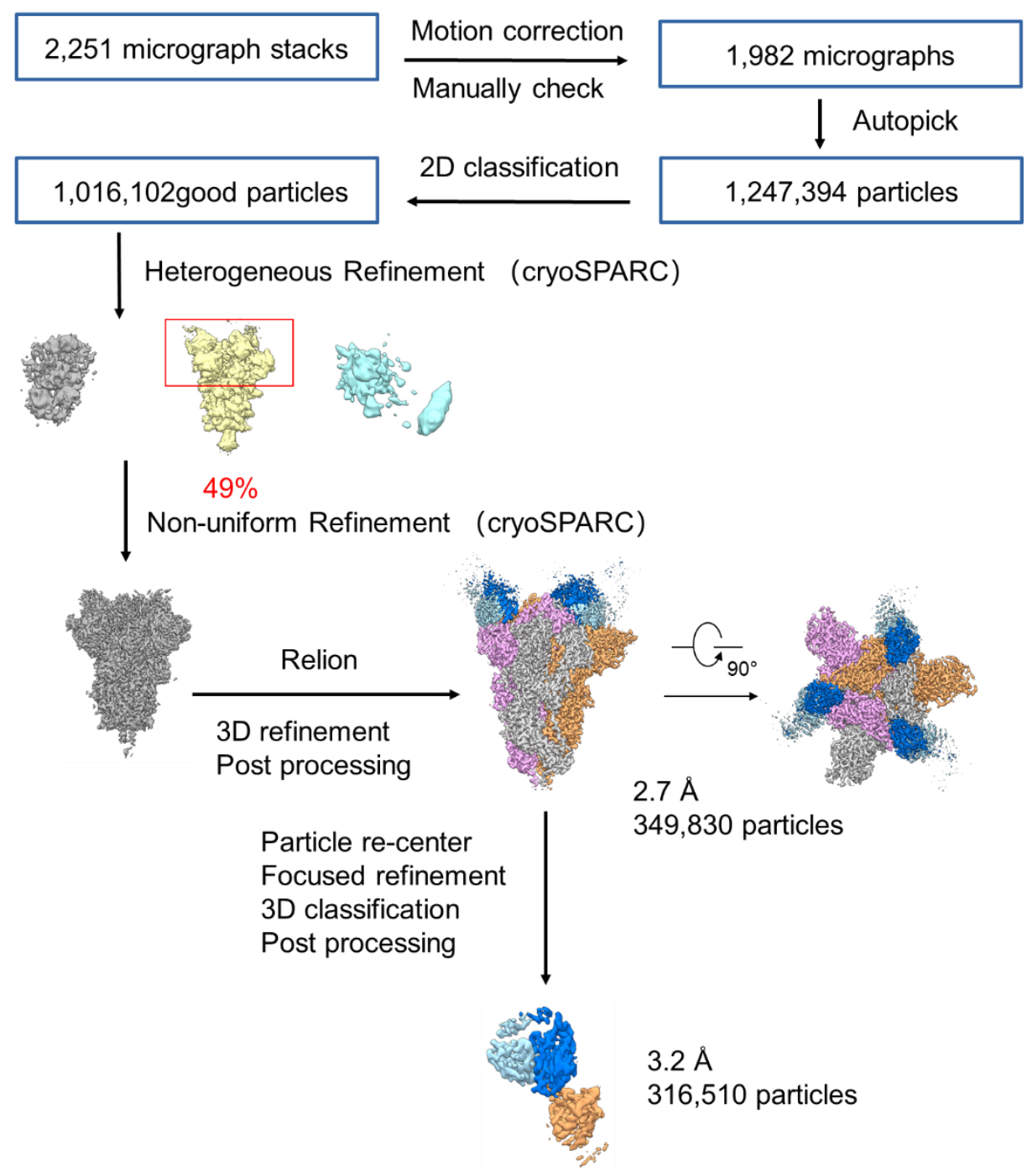

Fig. S7 Flowchart for cryo-EM data processing of SARS-CoV-2 S trimer in complex with 2G1. a, Representative cryo-EM micrograph and 2D class averages of cryo-EM particle images of SARS-CoV-2 S trimer bound with 2G1. The scale bar in 2D class averages is $10 \mathrm{~nm}$. b, Please refer to the 'Data Processing' in Methods section for details. 
Table S1 Data collection, 3D reconstruction and model statistic.

\begin{tabular}{|c|c|c|}
\hline Data collection & & \\
\hline EM equipment & \multicolumn{2}{|c|}{ Titan Krios (Thermo Fisher Scientific) } \\
\hline Voltage (kV) & \multicolumn{2}{|c|}{300} \\
\hline Detector & \multicolumn{2}{|c|}{ Gatan K3 Summit } \\
\hline Energy filter & \multicolumn{2}{|c|}{ Gatan GIF Quantum, 20 eV slit } \\
\hline Pixel size $(\AA)$ & \multicolumn{2}{|r|}{1.087} \\
\hline Electron dose (e-/Å2) & \multicolumn{2}{|r|}{50} \\
\hline Defocus range $(\mu \mathrm{m})$ & \multicolumn{2}{|r|}{$-1.2 \sim-2.2$} \\
\hline $\begin{array}{l}\text { Number of collected } \\
\text { micrographs }\end{array}$ & \multicolumn{2}{|r|}{2,251} \\
\hline $\begin{array}{l}\text { Number of selected } \\
\text { micrographs }\end{array}$ & \multicolumn{2}{|r|}{1,982} \\
\hline Sample & \multicolumn{2}{|c|}{ S protein in complex with $2 \mathrm{G} 1$} \\
\hline 3D Reconstruction & Whole model & $\begin{array}{l}\text { Interface between RBD and } \\
\qquad 2 \mathrm{G} 1\end{array}$ \\
\hline Software & $\begin{array}{l}\text { cryoSPARC/ } \\
\text { Relion }\end{array}$ & Relion \\
\hline Number of used particles & 349,830 & 316,510 \\
\hline Resolution $(\AA)$ & 2.7 & 3.2 \\
\hline Symmetry & \multicolumn{2}{|r|}{$\mathrm{C} 1$} \\
\hline Map sharpening $B$ factor $\left(\AA^{2}\right)$ & \multicolumn{2}{|r|}{-90} \\
\hline \multicolumn{3}{|l|}{ Refinement } \\
\hline Software & \multicolumn{2}{|r|}{ Phenix } \\
\hline Cell dimensions $(\AA)$ & \multicolumn{2}{|r|}{313.056} \\
\hline \multicolumn{3}{|l|}{ Model composition } \\
\hline Protein residues & \multicolumn{2}{|r|}{4,572} \\
\hline Side chains assigned & \multicolumn{2}{|r|}{4,572} \\
\hline Sugar & \multicolumn{2}{|r|}{78} \\
\hline Linoleic acid & \multicolumn{2}{|r|}{3} \\
\hline \multicolumn{3}{|l|}{ R.m.s deviations } \\
\hline Bonds length $(\AA)$ & \multicolumn{2}{|r|}{0.007} \\
\hline Bonds Angle $\left({ }^{\circ}\right)$ & \multicolumn{2}{|r|}{0.936} \\
\hline \multicolumn{3}{|l|}{$\begin{array}{l}\text { Ramachandran plot statistics } \\
(\%)\end{array}$} \\
\hline Preferred & \multicolumn{2}{|r|}{93.61} \\
\hline Allowed & \multicolumn{2}{|r|}{6.16} \\
\hline Outlier & \multicolumn{2}{|r|}{0.23} \\
\hline
\end{tabular}




\section{References}

9331 Volz, E. et al. Assessing transmissibility of SARS-CoV-2 lineage B.1.1.7 in England. Nature 593,

934 266-269 (2021).

9352 Alpert, T. et al. Early introductions and transmission of SARS-CoV-2 variant B.1.1.7 in the United

936 States. Cell 184, 2595-2604 (2021).

9373 Liu, J. et al. BNT162b2-elicited neutralization of B.1.617 and other SARS-CoV-2 variants. Nature

$938 \quad 596,273-275$ (2021).

9394 Dejnirattisai, W. et al. Antibody evasion by the P.1 strain of SARS-CoV-2. Cell 184, 2939$940 \quad$ 2954.e2939 (2021).

9415 Chen, R. E. et al. In vivo monoclonal antibody efficacy against SARS-CoV-2 variant strains. $942 \quad$ Nature 596, 103-108 (2021).

9436 Li, B. et al. Viral infection and transmission in a large, well-traced outbreak caused by the SARS944 CoV-2 Delta variant. medRxiv, doi:10.1101/2021.07.07.21260122 (2021).

9457 Andreano, E. \& Rappuoli, R. SARS-CoV-2 escaped natural immunity, raising questions about $946 \quad$ vaccines and therapies. Nat Med 27, 759-761 (2021).

9478 Cohen, A. A. et al. Mosaic nanoparticles elicit cross-reactive immune responses to zoonotic $948 \quad$ coronaviruses in mice. Science 371, 735-741 (2021).

9499 Ju, B. et al. Human neutralizing antibodies elicited by SARS-CoV-2 infection. Nature 584, 115950119 (2020).

95110 Lv, Z. et al. Structural basis for neutralization of SARS-CoV-2 and SARS-CoV by a potent 952 therapeutic antibody. Science 369, 1505-1509 (2020).

95311 Chi, X. et al. A neutralizing human antibody binds to the N-terminal domain of the Spike protein 954 of SARS-CoV-2. Science 369, 650-655 (2020).

95512 Liu, Z. M. et al. Identification of SARS-CoV-2 spike mutations that attenuate monoclonal and 956 serum antibody neutralization. Cell Host Microbe 29, 477-488 (2021).

Chen, R. E. et al. Resistance of SARS-CoV-2 variants to neutralization by monoclonal and serumderived polyclonal antibodies. Nat Med 27, 717-726 (2021).

Garcia-Beltran, W. F. et al. Multiple SARS-CoV-2 variants escape neutralization by vaccineinduced humoral immunity. Cell 184, 2372-2383 (2021).

Gupta, R. K. Will SARS-CoV-2 variants of concern affect the promise of vaccines? Nat Rev Immunol 21, 340-341 (2021). properties, virulence and epidemiology. Eur Rev Med Pharmacol Sci 25, 4405-4412 (2021). 202012/01 and other variants with spike deletion H69-V70, France, August to December 2020. Euro Surveill 26, 2100008 (2021).

8 Wang, P. F. et al. Antibody resistance of SARS-CoV-2 variants B.1.351 and B.1.1.7. Nature 593, 130-135 (2021).

Collier, D. A. et al. Sensitivity of SARS-CoV-2 B.1.1.7 to mRNA vaccine-elicited antibodies. Nature 593, 136-141 (2021). convalescent plasma. bioRxiv, doi:10.1101/2020.12.28.424451 (2020).

Madhi, S. A. et al. Efficacy of the ChAdOx1 nCoV-19 Covid-19 Vaccine against the B.1.351 Variant. N Engl J Med 384, 1885-1898 (2021). 
Zhou, D. et al. Evidence of escape of SARS-CoV-2 variant B.1.351 from natural and vaccineinduced sera. Cell 184, 2348-2361 (2021).

Alter, G. et al. Immunogenicity of Ad26.COV2.S vaccine against SARS-CoV-2 variants in humans. Nature 596, 268-272 (2021). Hoffmann, M. et al. SARS-CoV-2 variants B.1.351 and P.1 escape from neutralizing antibodies. Cell 184, 2384-2393 (2021).

Planas, D. et al. Reduced sensitivity of SARS-CoV-2 variant Delta to antibody neutralization. Nature 596, 276-280 (2021).

Augusto, G. et al. In vitro data suggest that Indian variant B.1.617 of SARS-CoV-2 escapes neutralization by both receptor affinity and immune evasion. Allergy, doi:10.1111/all.15065 (2021).

Liu, C. et al. Reduced neutralization of SARS-CoV-2 B.1.617 by vaccine and convalescent serum. Cell 184, 4220-4236 (2021). Padilla-Rojas, C. et al. Genomic analysis reveals a rapid spread and predominance of lambda (C.37) SARS-COV-2 lineage in Peru despite circulation of variants of concern. J Med Virol, doi:10.1002/jmv.27261 (2021). Laiton-Donato, K. et al. Characterization of the emerging B.1.621 variant of interest of SARSCoV-2. Infect Genet Evol 95, 105038 (2021).

Harvey, W. T. et al. SARS-CoV-2 variants, spike mutations and immune escape. Nat Rev Microbiol 19, 409-424, doi:10.1038/s41579-021-00573-0 (2021). Yuan, M. et al. Structural basis of a shared antibody response to SARS-CoV-2. Science 369, 11191123 (2020). protein. Science 370, 725-730, doi:10.1126/science.abd3255 (2020).

Walls, A. C. et al. Structure, Function, and Antigenicity of the SARS-CoV-2 Spike Glycoprotein. Cell 181, 281-292 (2020).

5 Cai, Y. F. et al. Structural basis for enhanced infectivity and immune evasion of SARS-CoV-2 variants. Science 373, 642-648 (2021).

Liu, Y. \& Rocklöv, J. The reproductive number of the Delta variant of SARS-CoV-2 is far higher compared to the ancestral SARS-CoV-2 virus. J Travel Med, doi:10.1093/jtm/taab124 (2021).

Hoffmann, M. et al. SARS-CoV-2 variant B.1.617 is resistant to bamlanivimab and evades antibodies induced by infection and vaccination. Cell Rep 36, 109415 (2021).

Lopez Bernal, J. et al. Effectiveness of Covid-19 Vaccines against the B.1.617.2 (Delta) Variant. N Engl J Med 385, 585-594 (2021).

39 Hsieh, C. L. et al. Structure-based design of prefusion-stabilized SARS-CoV-2 spikes. Science 369, 1501-1505 (2020).

Lei, J. \& Frank, J. Automated acquisition of cryo-electron micrographs for single particle reconstruction on an FEI Tecnai electron microscope. J Struct Biol 150, 69-80 (2005).

1 Zheng, S. Q. et al. MotionCor2: anisotropic correction of beam-induced motion for improved cryo-electron microscopy. Nat Methods 14, 331-332 (2017).

42 Grant, T. \& Grigorieff, N. Measuring the optimal exposure for single particle cryo-EM using a 
2.6 angstrom reconstruction of rotavirus VP6. Elife 4, e06980 (2015).

43 Zhang, K. Gctf: Real-time CTF determination and correction. Journal of Structural Biology 193, $1-12(2016)$.

44 Zivanov, J. et al. New tools for automated high-resolution cryo-EM structure determination in RELION-3. Elife 7, e42166 (2018).

45 Kimanius, D., Forsberg, B. \& Lindahl, E. Accelerated Cryo-EM Structure Determination with Parallelisation using GPUs in Relion-2. Elife 5, e18722 (2016).

46 Scheres, S. H. W. RELION: Implementation of a Bayesian approach to cryo-EM structure determination. Journal of Structural Biology 180, 519-530 (2012).

47 Scheres, S. H. W. A Bayesian View on Cryo-EM Structure Determination. J Mol Biol 415, 406418 (2012).

48 Punjani, A., Rubinstein, J. L., Fleet, D. J. \& Brubaker, M. A. cryoSPARC: algorithms for rapid 49 Rosenthal, P. B. \& Henderson, R. Optimal determination of particle orientation, absolute hand, and contrast loss in single-particle electron cryomicroscopy. J Mol Biol 333, 721-745 (2003).
Chen, S. X. et al. High-resolution noise substitution to measure overfitting and validate resolution in 3D structure determination by single particle electron cryomicroscopy. Ultramicroscopy 135, 24-35 (2013).

51 Trabuco, L. G., Villa, E., Mitra, K., Frank, J. \& Schulten, K. Flexible fitting of atomic structures into electron microscopy maps using molecular dynamics. Structure 16, 673-683 (2008).

52 Winn, M. D. et al. Overview of the CCP4 suite and current developments. Acta Crystallogr D 67,

53 Emsley, P., Lohkamp, B., Scott, W. G. \& Cowtan, K. Features and development of Coot. Acta Crystallographica Section D-Biological Crystallography 66, 486-501 (2010).

54 Adams, P. D. et al. PHENIX: a comprehensive Python-based system for macromolecular structure solution. Acta Crystallogr D Biol Crystallogr 66, 213-221 (2010). 